\title{
The bidirectional gut-brain-microbiota axis as a potential nexus between traumatic brain injury, inflammation, and disease.
}

\begin{abstract}
As head injuries and their sequelae have become an increasingly salient matter of public health, experts in the field have made great progress elucidating the biological processes occurring within the brain at the moment of injury and throughout the recovery thereafter. Given the extraordinary rate at which our collective knowledge of neurotrauma has grown, new insights may be revealed by examining the existing literature across disciplines with a new perspective. This article will aim to expand the scope of this rapidly evolving field of research beyond the confines of the central nervous system (CNS). Specifically, we will examine the extent to which the bidirectional influence of the gut-brain axis modulates the complex biological processes occurring at the time of traumatic brain injury (TBI) and over the days, months, and years that follow. In addition to local enteric signals originating in the gut, it is well accepted that gastrointestinal (GI) physiology is highly regulated by innervation from the CNS. Conversely, emerging data suggests that the function and health of the CNS is modulated by the interaction between 1) neurotransmitters, immune signaling, hormones, and neuropeptides produced in the gut, 2) the composition of the gut microbiota, and 3) integrity of the intestinal wall serving as a barrier to the external environment. Specific to TBI, existing pre-clinical data indicates that head injuries can cause structural and functional damage to the GI tract, but research directly investigating the neuronal consequences of this intestinal damage is lacking. Despite this void, the proposed mechanisms emanating from a damaged gut are closely implicated in the inflammatory processes known to promote neuropathology in the brain following TBI, which suggests the gut-brain axis may be a therapeutic target to reduce the risk of Chronic Traumatic Encephalopathy and other neurodegenerative diseases following TBI. To better appreciate how
\end{abstract}


various peripheral influences are implicated in the health of the CNS following TBI, this paper will also review the secondary biological injury mechanisms and the dynamic pathophysiological response to neurotrauma. Together, this review article will attempt to connect the dots to reveal novel insights into the bidirectional influence of the gut-brain axis and propose a conceptual model relevant to the recovery from TBI and subsequent risk for future neurological conditions.

Key words: Gut-brain axis, Traumatic Brain Injury, Concussion, Gut, Microbiota, Chronic Traumatic Encephalopathy, Neurodegenerative disease, Neuroinflammation, Microglia, Intestinal dysfunction 


\section{Introduction}

Affecting millions of individuals annually, traumatic brain injury (TBI) represents the leading cause of morbidity and mortality worldwide in individuals under the age of $45^{1}$. In addition to the acute symptoms following neurotraumatic events, there is increasing evidence that the long-term biological sequelae of these injurious events may increase the risk of neuropsychiatric disorders such as depression later in life while also contributing, in part, to the pathogenesis of neurodegenerative disease $^{2-5}$. In a recent report from the Centers for Disease and Control, TBI was labelled as a "silent epidemic" due to this rising prevalence, the common underreporting of injuries, and the often undetectable or undiagnosed neurobiological sequelae that may not clinically manifest until many years after the initial exposure to head trauma ${ }^{6,7}$. In congruence with this assertion from the CDC, the last decade of research has produced two important paradigm shifts in the understanding of TBI: 1) head trauma can incur damage to the brain's structure and function even in the absence of clinically evident TBI, and 2) head injuries should be treated as a disease process, not a static event ${ }^{8,9}$. This increased and deferred risk for future neurological disease following TBI suggests there is a large therapeutic window. Perhaps by attenuating the ongoing secondary biological injury mechanisms that persist following neurotrauma, it is possible to diminish the risk of neuropsychiatric illness and potentially mitigate the pathogenesis of neurodegenerative disease. In order to do so, it is important to examine both the local physiological processes promoting these disease processes as well as the 
peripheral influences occurring outside the confines of the CNS capable of exacerbating the local pathophysiological response and/or exert neuroprotective effects.

Concurrently to the increased attention to TBI, there has been a significant interest in investigating the role of the gut-brain axis in neurological health and disease. The gut-brain axis can be conceptualized as an integrative physiological model that incorporates afferent and efferent signals of neural, hormonal, and immunological origins, and dysfunction of this axis can have pathophysiological consequences ${ }^{10,11}$. Notably, the gut-brain axis is bidirectional with each entity capable of modulating the health of the other in either direction. The bidirectional influence of the gut-brain axis has been investigated for a growing variety of neuropsychiatric disorders, but how the composition of the gut modulates both the acute and chronic biological sequelae of neurotrauma is yet to be examined.

When examining the gut, it should be noted that recent trends in literature support the notion that the human body should be extended to that of the "human ecosystem"; or rather, the "human biome"12. This notion recognizes the mutualistic co-evolution of microbes and the human body. Recent estimates suggest that there are approximately $3 * 10^{13}$ bacterial cells that exist in every human body to comprise this ecosystem, a number which is thought to match or exceed the number of human cells ${ }^{13}$. The colon is most heavily colonized area of the human body with the concentration reaching approximately $10^{11}$ bacterial cells per gram in the colon ${ }^{13,14}$. While the entire functional significance of this microbial population is unknown, the composition and diversity of these commensal species inhabiting the gut can profoundly influence human physiology and, ultimately, the health of the human host across multiple physiological systems ${ }^{15,16}$. The central nervous system (CNS) is no 
exception as the composition of the gut microbiota is capable of influencing the development, function, and health of the $\mathrm{CNS}^{17}$. With this relatively new understanding, studies are increasingly probing the complex and varied interactions of the gut-brain-microbiota axis (GBMAx) ${ }^{18}$. While the broad influence of the microbiota on CNS health and function is increasingly appreciated by the scientific community, this is still a nascent field and the exact mechanisms are not fully understood ${ }^{19}$.

The gut microbiota, however, only comprises one aspect of the GBMAx, albeit an integral one. A more well understood, and perhaps more simplistic, component of the GBMAx relates to the function and integrity of the intestinal wall as a barrier to our external environment. It cannot be overlooked that the intestinal wall serves as the human body's largest interface with the surrounding environment as the combined mucosal surfaces of the gut are much larger than the more visible epidermal barrier ${ }^{20}$. This interface with the external environment has two critical functions, acting as 1) a filter with selective permeability to regulate the absorption of nutrients, electrolytes, and water, and 2) a barrier to prevent the penetration of pathogenic and harmful substances ${ }^{21}$. When the gastrointestinal (GI) tract is structurally compromised the functional integrity of this barrier is known to deteriorate. When this occurs, intestinal permeability increases and substances can aberrantly penetrate the barrier to disrupt normal physiological functions. Such deficits in intestinal permeability typically lead to an innate immune response that underpins chronically elevated levels of inflammation which are known to promote disease ${ }^{20,22}$. Of note, the gut microbiota likely plays a critical role in regulating intestinal barrier function ${ }^{23}$. Also of note, intestinal barrier dysfunction has been recognized as a consequence of $\mathrm{TBI}^{24-26}$. However, it remains unclear how the GBMAx influences recovery from TBI and the potential implications for subsequent health of the CNS. 
In this article, we explore the bidirectional involvement of the GBMAx following TBI. Specifically, based on published pre-clinical, clinical, and epidemiological data, we propose a theoretical framework that highlights the potential mechanisms by which the GBMAx may peripherally influence recovery from TBI and the subsequent risk of latent neurological disease. To provide a comprehensive picture of GBMAx involvement, the scope of this article will extend beyond the broad implications of gut microbiota alterations to also include the more distinct influence of intestinal barrier dysfunction. The proposed framework revolves around the current evidence highlighted in the reviews below that:

1) neurotrauma results in local inflammation within the brain, which induces microglial priming ${ }^{27}$,

2) primed microglia increase the brain's vulnerability to exaggerated immune responses to future external (e.g., subsequent TBI) or internal (e.g., infection) insults ${ }^{28}$,

3) if left unchecked, these hyperactive immune cells in the brain may become neurotoxic and trigger the pathophysiological cascade of various neurological conditions (e.g., depression, Alzheimer's, etc.) ${ }^{29}$,

4) and therefore, perpetual neuroinflammation increases the risk of neuropsychiatric and neurodegenerative disease ${ }^{30,31}$.

5) By looking beyond the confines of the CNS and incorporating the gut, it is clear that systemic and peripheral influences occurring outside the CNS can exacerbate the local pathophysiological response to TBI occurring within the brain, including ongoing neuroinflammation ${ }^{32}$.

6) TBI can cause structural and functional damage to GI tract ${ }^{24}$, 
7) this type of intestinal barrier dysfunction typically leads to increased intestinal permeability $^{33}$,

8) increased intestinal permeability is associated with a systemic immune response ${ }^{34}$, and

9) the systemic inflammatory response emanating from the damaged gut will exert influence on the vulnerable and previously primed microglia to further exacerbate neuroinflammation ${ }^{14,35,36}$.

\section{Background}

\subsection{New perspectives for TBI}

Most commonly, TBI is generally defined as a closed head injury as a result of acceleration/deceleration forces, and it is traditionally classified into three categories: mild, moderate, and severe, based on the Glasgow Coma Scale (GCS), which scores the patient's level of consciousness $^{37}$. Notably, $80-90 \%$ of head injuries can be classified as mild TBI and the majority of these injuries occur in the absence of LOC, which leaves only more subtle signs and symptoms available for diagnosis ${ }^{38}$. Despite this simplistic and traditional classification system, TBIs are remarkably heterogeneous. While the majority of mild TBI patients recover within days or weeks, it is estimated that symptoms persist for at least 3 months in $\sim 30 \%$ of patients and up to one year postinjury in $\sim 10 \%$ among patients suffering from this mild $\mathrm{TBI}^{39}$. Ambiguity surrounding the terminology and classification of neurotrauma has been the subject of additional scrutiny in part due to the many studies reporting the consequences of "subconcussive" hits to the head". Here, "subconcussive" refers to neurotrauma that does not result in clinically evident signs of head injury, yet may still provoke an occult neurobiological response ${ }^{40}$. Certain subpopulations such as members of the armed forces and athletes participating in contact sports are more frequently exposed to such 
neurotraumatic forces, both concussive and subconcussive. For example, studies of collegiate American football players equipped with accelerometers in their helmets found that athletes can sustain well over 1000 hits to the head in a single season ${ }^{41}$. This repetitive exposure can induce significant changes to the structure and function of the brain, even if each of the traumatic insults is deemed subconcussive in the absence of a clinically evident head injury ${ }^{42-45}$. While it has yet to be fully elucidated, this data suggests it is possible that damage to the brain can accumulate over time, and that the secondary biological injury mechanisms can be induced gradually without a singular concussive event.

This is relevant given the mounting evidence that prompted the recent paradigm shift to view TBI as a disease process rather than a static injurious event ${ }^{9}$. Literature suggests that the neuronal damage consequential to TBI is not exclusively induced by primary injury mechanism of mechanism of mechanical tearing/shearing and therefore, is not limited to static moment of the trauma. Rather, emerging evidence supports that much of the long-term axonal damage may be attributable to secondary biological injury mechanisms that are progressive and can persist long after the initial mechanical forces ${ }^{46,47}$. Even in cases of a single TBI, studies examining axonal pathology report that deleterious effects do not merely persist, but actually progress and continue to worsen for weeks, months, and years after the primary mechanical injury ${ }^{46}$. This effect is exacerbated in individuals who are exposed to repetitive head trauma, even when these blows to the head are deemed to be subconcussive $^{48}$. With this consideration, the pathophysiology of TBI should no longer be considered an irreversible outcome of a static injury event. Rather, there is likely a large therapeutic window to curb this ongoing injury process in order to mitigate chronic damage to the brain and reduce the 
otherwise heightened risk of neurological conditions, including depression and degenerative diseases such as Chronic Traumatic Encephalopathy (CTE).

The following section will provide an overview of this biological process with an emphasis on the neuroinflammatory response since this appears to be at risk of becoming chronically hyperactive following head injuries and is closely related to peripheral influences from the $\operatorname{gut}^{28,49}$.

\subsubsection{Immunological perspectives of TBI sequelae}

Glial cells outnumber neuronal cells by approximately 10 to 1 in the brain ${ }^{50}$. Among these glial cells are microglia, which are the primary immune cells of the CNS and are chiefly involved in the immune response to neurotraumatic events. The inflammatory response following TBI is nuanced; there are both beneficial and detrimental effects, which likely differ in the acute and chronic phases of recovery following the injury ${ }^{27,49,51}$. Generally, the immune system must respond appropriately to the primary injury, but it must do so transiently and in a regulated manner ${ }^{52}$. Secondary injuries mediated by biological mechanisms occur when the CNS immune response becomes dysregulated and shifts to a perpetual state of neuroinflammation ${ }^{53,54}$. These feedforward loops of perpetual inflammation, which may be triggered by TBI, increase the risk for neuropsychiatric illness and neurodegenerative disease that may not be clinically evident until many years after the initial injury ${ }^{54}$.

Chronic neuroinflammation has been found to persist years after neurotrauma in both experimental animal models of $\mathrm{TBI}^{55-58}$ and in human studies ${ }^{59-61}$. Relative to healthy controls, chronically elevated levels of neuroinflammation, as detected via PET scan, can persists both years after a single remote TBI as well as years after exposure to repetitive neurotrauama, even in the 
absence of a clinically evident $\mathrm{TBI}^{61-64}$. Notably, one study that also investigated neuronal white matter integrity found that levels of microglial activations correlated with structural changes in the brain $^{63}$. Recent reviews detailing this dysregulated immune response in the brain following neurotrauma highlight the phenomenon of microglial priming as a key component of this harmful pro-inflammatory state ${ }^{28,29,49}$. In short, priming refers to microglia that are in a hypersensitive and hyperactive state; they are "primed" for a future exaggerated response to an internal or external challenge. Given their hypersensitivity, these primed microglia require a lower stimulus to elicit a toxic pro-inflammatory response to future injuries, stressors, and infections while their hyperactivity results in an exaggerated inflammatory response to what might otherwise be regarded as a modest secondary immune challenge ${ }^{29,65}$. Subsequent exposure to neurotrauma (i.e. repetitive head injuries) is one apparent model for this phenomena as one head injury leaves the brain's microglia primed and more susceptible to becoming dysregulated with each subsequent hit ${ }^{28}$. However, non-traumatic peripheral influences can also pose an internal challenge that interacts with primed microglia to elicit a disproportionate inflammatory response in the brain.

To demonstrate the interaction between non-traumatic secondary peripheral challenges and primed microglia following neurotrauma, researchers administered systemic lipopolysaccharide (LPS; Box 1) challenges to mice that either received a head injury or sham-control event thirty days prior. Relative to the sham-controls, the mice that previously sustained an experimental head injury had a dysregulated immune response to the LPS challenge, which was exhibited by elevated levels of inflammatory cytokines (IL-1 $\beta$, TNF- $\alpha$, and IL-6) and morphological changes to microglia indicative of priming ${ }^{56,36}$. Additionally, the secondary LPS challenge caused depressive behavior only in mice that previously suffered head injuries and, thus, had primed microglia ${ }^{56}$. Notably, these depressive 
symptoms were not observable acutely following the head injury; they only emerged after the secondary immune challenge to the endotoxin thirty days post-injury. This indicates that 1) there is a large therapeutic window to harness and desensitize the immune system of the CNS, and 2) the brain may be susceptible to systemic peripheral immune challenges following TBI. The link between microglial priming and depression is further supported by studies devoid of head trauma entirely where repeated injections of inflammatory cytokines induce depressive symptoms only after microglia become primed ${ }^{66}$.

While the microglial polarization is the primary focus of this paper, there are many other neuroimmunological components involved in response to TBI. Among these processes includes the release of damage-associated molecular patterns (DAMPs), glutamate excitotoxicity, mitochondrial dysfunction, and the adaptive immune response mediated by $\mathrm{T}$ cells and B cells capable of utilizing the lymphatic and glympahatic systems. As Simon et al explain in their comprehensive review, all of these processes have the potential to become pathogenic ${ }^{67}$.

\section{Box 1: Key Points Related to Lipopolysaccharide (LPS)}

- $\quad$ LPS is a bacterial endotoxin known to provoke an innate immune response ${ }^{68}$

- LPS injection (aka experimental endotoxemia) is commonly used by researchers to induce a systemic immune response in human subjects ${ }^{69}$ and experimental animal models ${ }^{70}$

- In healthy adults, experimental endotoxemia is shown to robustly activate microglia indicative of neuroinflammation ${ }^{69}$

- Acutely, LPS-mediated neuroinflammation causes "sickness behavior", including depressive symptoms ${ }^{71}$

- Experimental endotoxemia is used as a model to induce neurodegeneration in preclinical studies ${ }^{70}$

- During intestinal barrier dysfunction (i.e. increased permeability), endogenous LPS can "leak" across defective tight junctions of the intestines into the body's circulation, which results in an immune response that can spur neuroinflammation and neuropsychiatric illness ${ }^{72}$

- LPS can disturb previously primed microglia to exacerbate neuroinflammation and prompt disease ${ }^{56}$

- Serum LPS levels emanating from damaged intestinal walls found to correlate with disease severity for neurodegenerative disorders in human patients (e.g., Parkinson's) ${ }^{73}$ 


\subsection{GBMAx: Linking the gut and neuroinflammation}

Peripheral influences are increasingly recognized as key regulators of central physiological processes within the brain during both health and disease ${ }^{74}$. Chief among these peripheral stimuli are signals emerging from the gut to comprise the bidirectional gut-brain axis ${ }^{11,19,75,76}$.

As introduced earlier, the gut microbiota is principally involved in the bidirectional influence of the GBMAx. Notably, the collective genome of the $10^{13}$ microorganisms inhabiting the gut, termed the microbiome, is both larger and more plastic than that of the human genome $\mathrm{in}^{13,77,78}$. The gut microbiota is partially inherited from the mother at birth but, in varying degrees, many environmental factors influence the composition of the gut microbiota such as mode of birth delivery, antibiotic consumption, and $\operatorname{diet}^{79-81}$. Despite inconclusive findings, clinical data also suggests that the composition of the gut may be altered in the presence of disease, including those considered neurological. Endophenotypes have been observed linking microbial dysbiosis to an expanding list of CNS conditions that includes autism spectrum disorder, depression, and Parkinson's disease ${ }^{82}$. Though it remains difficult to infer causality from these correlational observations, these findings further highlight the plastic nature of the gut microbiota and its potential implications with neuronal health.

Strong evidence from these preclinical models suggest that the GBMAx is bidirectional; mental state (e.g., stress) can alter the gut microbiota while the gut microbiota is also capable of exerting influence on the $\mathrm{CNS}^{19}$. Preclinical evidence demonstrating the modulatory effects of gut microbiota on the brain are primarily derived from 1) "germ-free" models where diseases are studied in animals devoid of microbial species, 2) models in which microbial manipulation is accomplished with antibiotics, 
and 3) models of fecal microbiota transplantation (FMT) ${ }^{79,83-85}$. When examining this bottom-up influence of the GBMAx, evidence suggests the gut microbiota is mechanistically important for the regulation of central CNS processes. Commensal species in the gut can signal the brain to modulate processes such as neurotransmission, neurogenesis, microglial activation, and influence behavior under both homeostatic conditions and in response to internal/external stressors ${ }^{19,86,87}$. Evidence also suggests that these commensal species may control the development and function of immune cells throughout the body, including the microglia in the $\operatorname{brain}^{88-90}$. Therefore, the interaction between gut microbiota, neuroinflammation, and disease may be implicated as a peripheral influence for the pathogenesis of neurological disease ${ }^{91-93}$. While it is difficult to infer causality from available data, recent studies performing FMTs from clinical patient populations (e.g., depression, PD) to murine species provide compelling evidence as mice receiving FMTs from healthy humans remain healthy, while those who receiving FMTs from clinical patients begin to exhibit symptoms characteristic of the disease of the human FMT donor ${ }^{75,94}$.

Given the existing evidence suggesting that gut microbiota can influence neurological health through mechanisms that may be mediated by inflammation, this is certainly a compelling target for future research. However, gut barrier dysfunction may arguably be a more distinct source of systemic inflammation that ultimately affects the brain, though this is not entirely independent of the gut microbiota. When this barrier is structurally compromised and intestinal permeability increases, pathogenic substances such as LPS (Box 1) can aberrantly penetrate the barrier. This provokes an innate immune response that causes systemic inflammation while also peripherally triggering or exacerbating ongoing neuroinflammation. In support of this association between gut barrier dysfunction and the subsequent LPS-mediated neuroinflammation, a recent clinical trial used PET 
scans to detect robust microglial activation following peripheral LPS injection in healthy adults ${ }^{95}$. Given our previous discussion of microglial priming, intestinal dysfunction resulting in endotoxemia may increase one's susceptibility to neurological disease; especially amongst those with history of TBI. To this end, pre-clinical evidence indicates that serum LPS levels correlate with neurodegenerative pathology and neuronal cell death ${ }^{35,91,96-98}$. This is further supported by clinical data as studies report that circulating levels of serum LPS strongly correlate with disease severity in AD, PD, and ALS patients ${ }^{73,99}$. Notably, levels of serum LPS in patients with neurodegenerative disease correlate with discrete measures of intestinal permeability, which suggests that gut barrier dysfunction is the source of this endotoxemia ${ }^{73}$. The notion of LPS mediated neurodegeneration is so robust that peripheral LPS injections are commonly used as a model for intentionally inducing neurodegenerative disease in pre-clinical experiments ${ }^{100,101}$.

Therefore, it is essential to maintain the health and structural integrity of the intestinal mucosa. Indeed, the intestinal barrier is compromised in many disease states, but the resilience of the gut may be compromised in disease free individuals as well due to nutrient deficiencies ${ }^{20}$. Dietary fiber, for example, is arguably the most important nutrient to maintain a healthy, resilient intestinal barrier ${ }^{102}$. Dietary fiber is technically indigestible; upon reaching the colon, it is fermented by gut microbiota. When fiber is depleted and there are inadequate nutrients for the gut microbiota to self-sustain, the epithelial tissue lining the gut becomes damaged which inherently compromises the functional integrity of the barrier ${ }^{102}$. Remarkably, the National Health and Nutrition Examination Survey (NHANES) data indicates that less than 5\% of Americans across all gender and age subgroups achieve the recommended guidelines for adequate intake of dietary fiber ${ }^{103}$. This suggests that the average American has suboptimal health of the epithelial tissue comprising the GI tract, which may precipitate 
future permeability deficits ${ }^{33}$. In addition to maintaining structural integrity of the intestinal wall, dietary fiber intake regulates inflammation through the production of SCFAs as a metabolic byproduct of microbial fermentation ${ }^{104}$.

As further described in the conceptual model below, the structural integrity and resilience of the intestinal membrane may be a crucial component to one's neurological health following neurotraumatic events.

\section{Exploring the Bidirectional GBMAx Involvement following TBI}

\subsection{Top-Down: Brain-to-Gut}

A recent review explored the extent to which the brain influences the gut following TBI with primary focus on GI dysfunction following $\mathrm{TBI}^{24}$. To function properly, the GI tract and other visceral organs rely on innervation from the enteric nervous system, which is a component of the autonomic nervous system and largely mediated by vagal pathways. As Kharrazian highlights in this review, brain injuries often cause autonomic imbalances collectively referred to as dysautonomia ${ }^{24}$. This disruption of the autonomic nervous system following TBI is a common symptom with $92 \%$ of patients exhibiting significantly altered parameters of autonomic function in the first seven days post-severe $\mathrm{TBI}^{105}$. The incidence of overt symptoms arising from dysautonomia appear to be reduced over time, but the findings from a separate case-control study suggest that these symptoms may be a chronic consequence of $\mathrm{TBI}^{106}$.

One major characterization of TBI induced dysautonomia is GI dysfunction ${ }^{107}$. Remarkably, head trauma can induce both structural and functional changes to the epithelial tissue lining the gut. 
Functionally, decreased contractility of the smooth muscle within the GI tract results in delayed transit times following $\mathrm{TBI}^{108,109}$. Pre-clinical data suggests that this functional impairment is correlated with both increased systemic inflammation in the gut and brain atrophy ${ }^{108}$. In addition to decreased intestinal contractility, experimental neurotrauma can trigger alterations to the intestinal mucosa morphology and significant structural damage to the tissue lining the GI $\operatorname{tract}^{25}$. This structural damage to the intestinal barrier is evident as early as three hours post-injury, it peaked at 72 hours, and the damage was still evident at the study's conclusion seven days post-injury ${ }^{25}$. While the exact mechanisms are unknown, evidence suggests that damage to the GI tract following TBI is mediated by defective tight junctions in the intestinal wall ${ }^{26,110}$. Other observed morphological alterations of the gut mucosa occurring within three hours of TBI include shedding of epithelial cells, fracture of intestinal villi, fusion of adjacent villi, focal ulcer, dilation of central chyle duct, mucosal atrophy, edema in the villous interstitium and lamina propria, and a reduced expression of intestinal tight junction proteins (e.g., occludin, ZO-1 $)^{25,26}$.

Taken together, alterations to the gut's structure and function following head injuries leads to increased intestinal permeability and gut barrier dysfunction ${ }^{25,26,111-114}$. Aberrant intestinal permeability results in endotoxemia that prompts significantly greater levels of inflammation within the gut ${ }^{115-117}$. Elevated levels of CD40 expression, NF-k $\beta$, TNF- $\alpha$, IL- 6 and other inflammatory cytokines in response to increased intestinal permeability can trigger or exacerbate a vicious proinflammatory cascade effecting systems outside the gut, including the CNS. Importantly, measures of intestinal permeability (e.g., lactulose/mannitol ratio) correlate with both levels of plasma endotoxin and inflammation at all time points following experimental $\mathrm{TBI}^{25}$. Also of note, pre-clinical data indicates that bacterial translocation following TBI is only evident within the intestine and 
bacterial colonies are not detectable in the lung, liver, spleen, or kidney when measured at 7 days post-injury ${ }^{118}$. This same study reports that interventions supporting intestinal barrier function can rescue intestinal permeability deficits to prevent bacterial translocation ${ }^{118}$. Whileore research is this suggests that intestinal dysfunction is mechanistically relevant for the post-TBI endotoxemia and resultant systemic inflammatory response.

In the context of mechanical TBI, there is limited data showing the "top-down" effects of head injury on gut microbiota alterations. In their experimental weight-drop model, Houlden et al. observed TBI induced dysbiosis in mice. Specifically, they report that TBI severity correlated with changes in Bacteroidetes, Porphyromonadaceae, Firmicutes, and Proteobacteria ${ }^{119}$. More insights can be gained by investigating alternative mechanisms of acute brain injury (See: Section 3.3).

\section{2. "Bottom-Up": Potential Gut-to-Brain Signaling}

Considering the effects of neurotrauma on the structure and function of the gut, it is necessary to examine the potential influence of the GBMAx in the opposite direction; how does neurotrauma induced damage to the gut affect the brain? Given the local immunological aspects of the sequelae following TBI within the CNS, could gut barrier dysfunction and/or microbiota alterations spur a peripheral inflammatory cascade that further exacerbates the dysregulated hyperactivity of the microglia in the CNS initially perturbed by the primary insult?

While this has not been directly investigated, we can look to studies that induce experimental endotoxemia by peripherally injecting LPS, which effectively simulates TBI induced intestinal 
dysfunction. Even in healthy adults, trials consistently demonstrate that the LPS-mediated systemic inflammatory response is capable of peripherally modulating CNS health and function ${ }^{120,121}$. Collectively, the neurological symptoms following acute exposure to LPS challenge are referred to as "sickness behavior"" . Notably, the LPS-induced systemic inflammatory cytokines that are elevated by experimental endotoxemia and thought to provoke "sickness behavior" are the same inflammatory markers previously found to be predictive of a worse outcome following $\mathrm{TBI}^{122}$. Su and colleagues report that elevated levels of CRP, which is indicative of systemic inflammation, at 1, 2, and 3 months after a mild TBI was significantly correlated with persistent neuropsychological symptoms ${ }^{122}$. Of particular note, the typical symptoms of sickness behavior following injections of LPS in models of experimental endotoxemia shares considerable overlap with a constellation of symptoms often referred "post-concussion syndrome (PCS)" 71,123,124. In fact, Rathbone and colleagues recently made a compelling case to re-term PCS as "post-inflammatory brain syndrome" given this extensive overlap ${ }^{125}$. Perhaps the GBMAx is mechanistically involved in these persistent symptoms following neurotrauma in the subset of patients experiencing PCS. This was indirectly tested in a preclinical model where rats displayed significantly worsened outcome following an experimental head injury only upon exposure to a latent LPS injection ${ }^{116}$. The researchers attributed this secondary brain damage following the LPS exposure to persistent neuroinflammation, which may be occult in patients with damaged gut. Is it possible that the inflammatory cytokines released in response to peripheral endotoxemia can alter the activity of microglia in the brain to influence neuronal health following TBI?

There is evidence supporting that 1) locally, TBI induces neuroinflammation and primes microglia ${ }^{28}$; 2) even in healthy adults, peripheral LPS injection produces robust microglial activation ${ }^{95}$; and 3) 
hyperactive microglia predispose individuals to neurological disease ${ }^{31}$. Therefore, if head trauma causes intestinal barrier dysfunction, the resultant endotoxemia mediated peripheral inflammation can significantly influence the microglia that are already primed and vulnerable from the primary injury forces. In this conceptual model, we hypothesize that this may cause a harmful positive feedback loop as the microglia, which are already primed in response to the mechanically injured neuronal cells, may be aggravated and further dysregulated by the peripheral immune response originating in the gut (Figure 1). As a point of emphasis, this conceptual model has yet to be directly investigated.

\subsection{Closing the Loop: Insights from acute cerebral ischemic events}

While the primary context of the article relates to the pathophysiological mechanisms of TBI insights may be gathered from studies

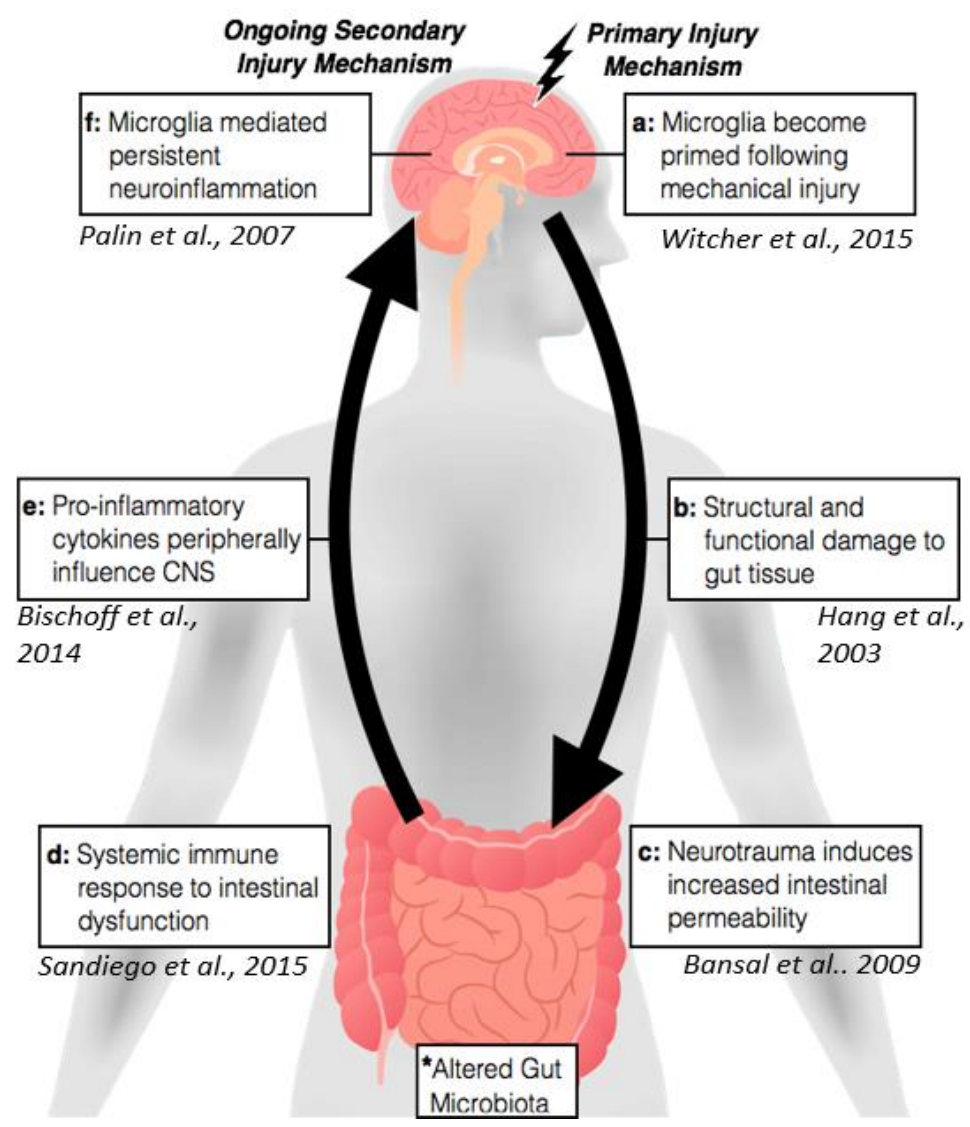

Figure 1: Proposed Conceptual Model for the Bidirectional Involvement of GBMAx following TBl: a) Neurotraumatic event occurs. Rotational and/or linear mechanical forces (e.g., acceleration/deceleration) of the event cause the "primary injury" to neuronal tissue (e.g., axonal shearing). Locally, neuroinflammation occurs as a consequence of this mechanical insult and microglia become primed. b) Neurotrauma can cause structural (e.g., defective tight gap junctions) and functional (e.g., decreased smooth muscle contractility) damage to the GI tract. c) Both the functional and structural changes to the intestinal membrane compromise the integrity of the intestinal wall barrier. This results in increased intestinal permeability. d) Increased intestinal permeability promotes a state of endogenous endotoxemia. Toxins (e.g., LPS) aberrantly permeating across the intestinal membrane trigger a systemic immune response with the release of pro-inflammatory cytokines (e.g., TNF-a, NFK-B, IL-6). e) Systemic release of pro-inflammatory cytokines peripherally influences the CNS. These inflammatory cytokines act on the microglia to stimulate a state of neuroinflammation. f) The microglia are previously primed from the local traumatic injury and, thus, are more vulnerable to future internal/external insults. Upon being peripherally stimulated by this systemic immune response in the gut, the microglial mediated neuroinflammation exacerbates the ongoing local secondary injury mechanisms.

*Pre-clinical data suggests that the gut microbiota are bidirectionally involved, but there is not enough evidence to elucidate the complex role of these commensal species 
investigating the bidirectional involvement of the GBMAx following other acute ischemic brain injuries $^{126-128}$.

Indeed, GI dysfunction is a known consequence of acute ischemic brain injuries ${ }^{129}$. Preclinical data from experimental models of ischemic stroke report direct evidence of intestinal barrier dysfunction, which leads to 'bacterial translocation"130-132. Bacterial translocation can be defined as the invasion of indigenous bacteria or endotoxins across the gut mucosa and into the circulation causing systemic inflammation and distant organ injury ${ }^{133}$. This is the same phenomena that we propose may be influencing outcome and risk of neurological disease following TBI. Across these various mechanisms of brain injury, future research is required to further elucidate the mechanisms mediating the subsequent intestinal permeability deficit. In a preclinical model of acute ischemic stroke, Stanley and colleagues observed that intestinal barrier dysfunction correlated with significant enteric neuronal loss in the submucosal plexus of the ileum, which they report may be mediated a misbalance between adrenergic and cholinergic signaling in post-stroke mice. Notably, the structural integrity of the gut barrier was observed at 8 different time points across a 24-hour time period post experimental injury, with intestinal permeability peaking at three hours post-injury ${ }^{130}$. There is also clinical data in support of these experimental findings suggesting stroke induced intestinal barrier dysfunction. In acute ischemic stroke patients, a biomarker for endotoxemia (Lipopolysaccharide Binding Protein; LBP) is not only significantly correlated with serum markers for inflammation, but LBP levels were also predictive of patients who were at highest risk of post-stroke infections ${ }^{134}$. Though there were no healthy controls in this study, stroke patients who eventually had an infection had significantly elevated levels of LBP relative to the "no infection" cohort of patients as early as 12 hours post-injury. 
In addition to gut-barrier dysfunction, studies of acute ischemic stroke provide further insight into the potential alterations to the composition of the gut microbiota and the implications of this dysbiosis. Houlden et al investigated the effects of neurotrauma on gut microflora with two separate experimental models for both closed head TBI and surgical ischemic brain injury ${ }^{119}$. This preliminary study reports neurotrauma induced alterations in gut microflora in both groups of mice, though they differed. In both the models of TBI and ischemic stroke, the bacterial population shifts correlated with both severity of the injury and neurological deficits. However, the specific microbial population shifts differed between the two models; the surgical ischemic brain injury produced significant shifts in Peptococcaceae (increased) and Prevotellaceae (decreased), which were both absent in the closedhead model of TBI. Notably, these microbial shifts correlated with lesion severity. The researchers note that these microbial alterations following neurotrauma may be mediated by goblet cells and the noradrenergic response of the autonomic nervous system. This study also emphasizes the potentially confounding effects of microbial population shifts following sham surgeries ${ }^{119}$.

To highlight the gut-to-brain influence of dysbiosis in acute brain injury, one experimental model artificially induced dysbiosis in mice with a two week course of antibiotics prior to surgical ischemic injury. Indeed, the microbial compositions of the gut significantly influenced outcome following stroke. This data suggests that the gut microflora is mechanistically involved in mediating the immune response to experimental brain injury, specifically through regulation of intestinal $\mathrm{T}$ cells ${ }^{135}$. The findings of Singh et al further demonstrate the bidirectional influence of the GBMAx following acute ischemic brain injury. To demonstrate this bottom-up effect that dysbiosis is causally linked to deteriorated stroke outcome, Singh et al utilized both germ-free mice and fecal microbiota transplants. There were three cohorts of mice: a) injured, b) sham, and c) germ-free \& injured. The microbiota 
colonized mice who received stroke became dysbiotic. When germ-free mice received fecal microbiota transplant from the dysbiotic post-stroke mice, it exacerbated lesion volume and functional deficits. Alternatively, recolonization with a transplant of the normal sham-control microbiota improved stroke outcome in both cohorts of injured mice while also normalizing the lesion-induced dysbiosis ${ }^{136}$. Notably, the researchers suggest that the gut microbiota exert influence through modulating the neuroinflammatory response. In another experimental model, researchers report that artificially depleting gut microbiota with broad-spectrum antibiotics pre-ischemic injury is associated with a significantly worsened outcome ${ }^{137}$. Alternatively, pre-clinical data also indicates that modulating the gut microbiota with probiotics is neuroprotective as behavioral deficits were rescued following cerebral ischemia/reperfusion injury ${ }^{138}$. This data has immediate clinical relevance considering the high-rates of antibiotic use in order to combat post-stroke infections.

There is also emerging clinical data to further support these preliminary experimental findings. To date, these clinical studies are observational in nature and report on the "top-down" implications of the GBMAx following stroke. Swidsinski et al were amongst the first researchers to report a significant dysbiotic shift in the gut microbiota amongst stroke patients. The researchers note that the dysbiotic response to ischemic brain injury is worse than what is typically observed in patients with ulcerative clolitis patients, suggesting severe GI dysfunction ${ }^{139}$. This was supported by a case-control study that observed significant gut dysbiosis following ischemic brain injury when compared to asymptomatic age-matched controls with atherosclerosis ${ }^{140}$. Most recently, a study found that dysbiotic alterations correlated with markers of systemic inflammation (e.g., IL-6, CRP) following stroke $^{141}$. Notably, causality cannot be inferred from this clinical data since there are no pre-stroke 
measures of gut microflora in these patients. Future research is required in this emergent field to more comprehensively elucidate the GBMAx involvement in brain injury patients.

\section{Discussion and Future Directions}

\subsection{Potential Clinical Implications}

As previously discussed, heightened levels of systemic inflammatory markers are indicative of worse outcomes following TBI, even those considered mild ${ }^{122}$. Therefore, in this conceptual model, we postulate that the GBMAx may be a therapeutic target to attenuate this systemic inflammatory response to augment recovery in the acute and/or subacute phases following the head injury (Figure 2). In this context, the GBMAx may serve as a bidirectional loop of inflammation with primed microglia that are being further provoked by peripheral inflammatory stimuli originating in the gut. Alternatively, this model may more aptly apply to stroke and severe TBI patients as a means to mitigate the risk for infection that is otherwise heightened due to bacterial translocation and/or separate mechanisms of immunosuppression, which may have immediate clinical potential for stroke patients. Despite the debilitating neurological deficits and injury to neuronal tissue, infection, namely bacterial pneumonia, is considered the leading cause of death after stroke ${ }^{142}$. Recent evidence suggests the bacteria responsible for these potentially lethal infections following stroke emanate from the gut ${ }^{130}$. Perhaps this is, in part, caused by intestinal barrier dysfunction whereby indigenous microbial species aberrantly penetrate the compromised barrier? 


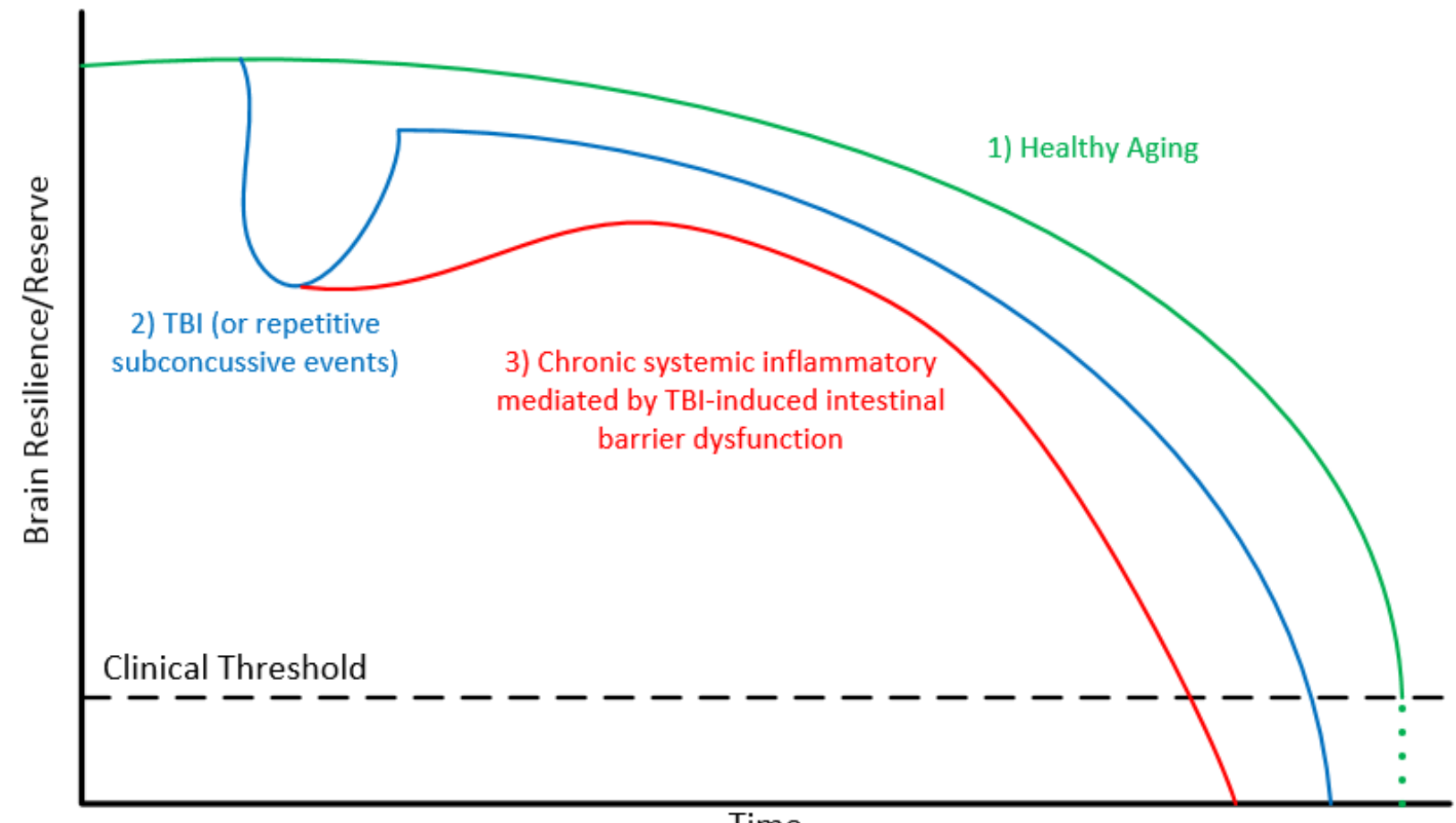

Time

Figure 2: This figure illustrates the hypothetical acute/subacute implications of this proposed model. 1) In healthy aging, the brain becomes naturally more susceptible to neurological disease (green line). 2) The brain typically recovers from TBI, but is now less resilient as TBI is a risk factor for numerous neurological conditions (blue line). 3) If the GBMAx is involved acutely following TBI, there may be TBI induced dysbiosis and/or intestinal dysfunction (red line). The resultant systemic inflammatory response can impair recovery and the brain will be more susceptible to neurological disease. Preclinical and clinical data to date support that systemic inflammation following TBI is associated with worse outcomes. Preclinical data reviewed in Section 3 of this manuscript further demonstrates that the GBMAx may be a source of this systemic inflammatory response.

When considering the long term implications of the proposed model, it is notable that multiple lines of evidence support the pathogenic role of inflammation in both neuropsychiatric illness and neurodegenerative disease $\mathrm{s}^{31,101,143}$. There are experimental models highlighting the correlation between ongoing neuroinflammation in both neuropsychiatric symptomology and in the progression of neurodegenerative pathology ${ }^{144-154}$. In clinical data, there are observational findings of elevated levels of neuroinflammation in CNS conditions ranging from depression to Alzheimer's disease ${ }^{30,155-}$ 
161. Further, longitudinal epidemiological data suggests that chronic systemic inflammation often precedes the onset of these conditions and therefore, is a likely risk factor ${ }^{162-164}$. This interaction between neurotrauma, microglial priming, and systemic inflammation is likely involved in the mechanisms that predispose individuals to neurological conditions in the years that follow $\mathrm{TBI}^{54,165-}$ 168. With this consideration, we believe it is crucial to harness the systemic immune response to attenuate these disease processes, which may be mediated by GBMAx.

The concept of microglial priming is crucially involved in the proposed model when considering this latent influence of the GBMAx on the susceptibility to neurological disease. The capacity for microglia to stay regulated can be conceptualized as a key component to the brain's resilience against future neurological disease ${ }^{169,170}$. When microglia become primed, as they do following TBI, the immune system of the CNS becomes less resilient to future internal or external stressors. We propose that isolated and remote events GBMAx dysfunction later in life could be a source of internal stressors that provoke the previously primed microglia to further predispose individuals to disease(Figure 3). 


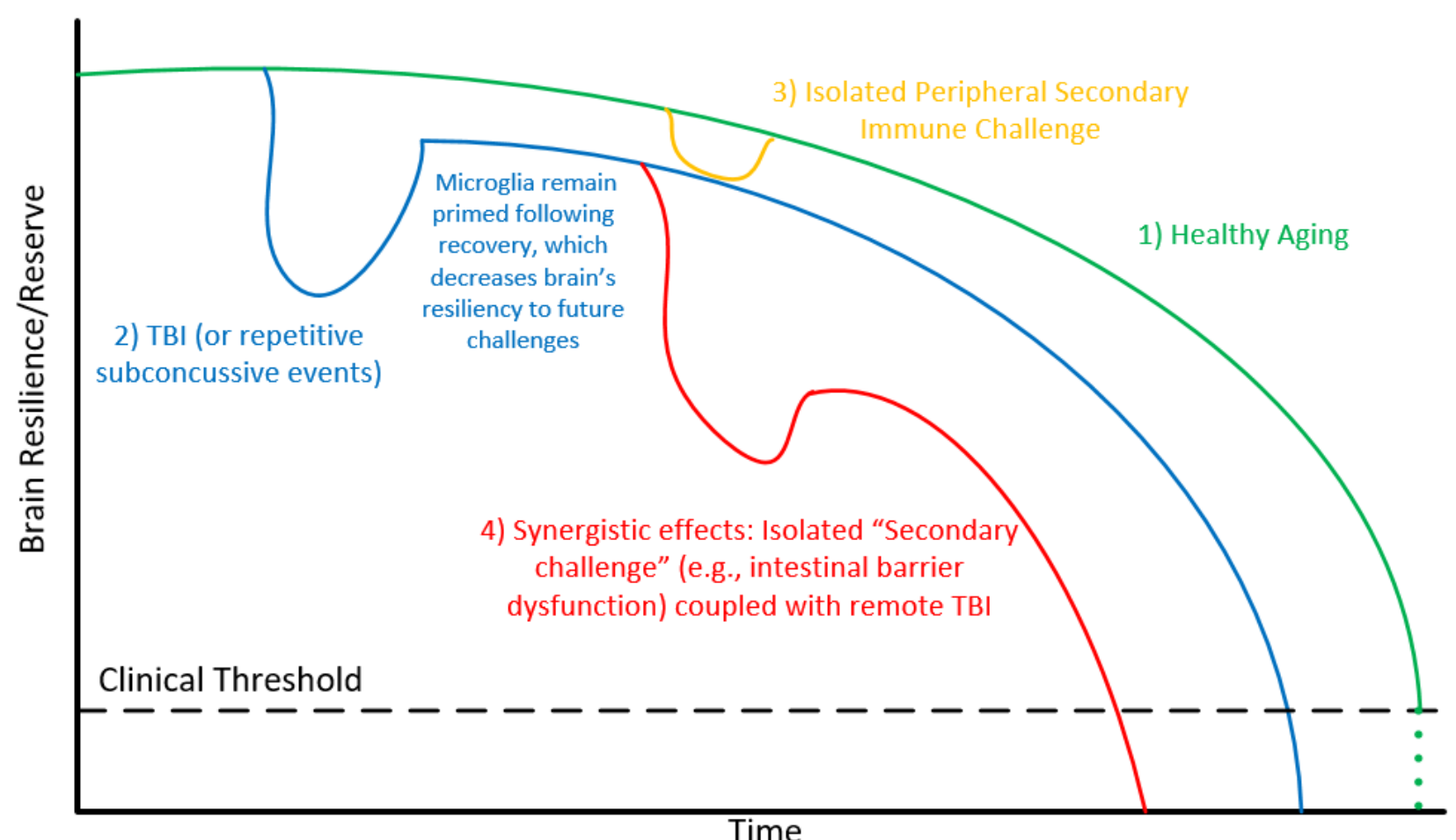

Figure 3: This figure illustrates the hypothetical long-term implications of the proposed model. 1) In healthy aging, the brain remains resilient yet becomes naturally more susceptible to neurological disease over time (green line). 2) The brain typically recovers from TBI, but is less resilient to future insults. This decreased resiliency is partially mediated by the microglial priming that occurs and persists following the TBI. This increased risk for latent neurological disease following TBI is well supported in the clinical data (blue line). 3) In the absence of TBI, a secondary peripheral immune challenge does not provoke a sustained pathological response, which was demonstrated in a pre-clinical model of experimental endotoxemia following TBI (Fenn et al. 2014) (orange line). 4) The resiliency deficit from the initial TBI leaves the brain susceptible to sustained neurological disease when exposed to secondary immune challenges (Fenn et al 2014). The GBMAx, even if not directly affected by the initial injury, could latently influence neurological health following TBI. Any future GBMAx disruption leading to gut dysbiosis and/or intestinal barrier dysfunction could spark a secondary immune challenge that the brain is unable to withstand due to the previously primed microglia that increase susceptibility to disease. Such GBMAx disruptions include antibiotic induced dysbiosis, Inflammatory Bowel Disease, Irritable Bowel Syndrome, stress, poor diet, etc. Therefore, per this hypothesis, therapeutic intervention that fortify the intestinal barrier may have a neuroprotective effect against latent neurological disease (red line).

There are numerous and varied examples of isolated GBMAx disruptions independent of TBI that could cause intestinal barrier dysfunction and/or gut dysbiosis such as 1) mental stresss ${ }^{14}, 2$ ) 
inflammatory bowel disease, which is increasingly prevalent $\left.{ }^{171}, 3\right)$ antibiotic induced dysbiosis, and 4) even the poor Western Diet of the average American is reported to promote dysbiosis and impair intestinal barrier function ${ }^{172}$. This may be a greater concern as individuals age because the aging process influences the activity of these glial cells in a manner that further shifts microglia towards the 'primed' phenotype, which we hypothesize decreases the brain's resilience ${ }^{173}$. This hypothesis is similar to that of other research groups who have postulated that TBI is lowering the brain's resilience (or 'cognitive reserve') through these microglial-mediated mechanisms ${ }^{53,54,168,174}$. Given the extensive literature reviewed in this article, we believe it is necessary to consider the influence of the GBMAx as a source of secondary insults that exacerbates neuroinflammation to increase susceptibility to disease.

Future research is needed, but we hypothesize that increasing the "resilience" of gut health may have neuroprotective effects and mitigate the risk of latent neurological disease by reducing the chance of an independent secondary peripheral challenge.

\subsection{Potential Therapeutic Strategies}

This integrative understanding of the pathophysiology of head injuries opens the door to novel therapeutic approaches with the potential to improve patient outcomes and mitigate risk for the onset of latent neurological diseases. This nascent field of research offers many potential avenues to further investigate the influence of the GBMAx to halt secondary injury mechanisms from continuing to cause insidious damage following neurotrauma.

\subsubsection{Targeting the Microbiota}


The plastic nature of the human microbiome makes it a potential therapeutic target, and probiotic supplementation is one avenue to alter the composition of the gut microflora. One mechanism by which probiotics may influence the pathophysiological response to head injuries is through regulating intestinal permeability ${ }^{175}$. Mechanistically, probiotics generally work to tighten gap junctions ${ }^{176}$, which are often a source of disruption following TBI. In fact, existing preclinical data provides direct evidence in support of this in a model where probiotic supplementation after the neurotrauma significantly lowered the intestinal permeability that was otherwise increased by the damage to the intestinal mucosa following $\mathrm{TBI}^{118}$. These findings suggest that probiotics can fortify the intestinal barrier to mitigate neurotrauma induced endotoxemia and inhibit systemic dysregulation of the immune system that may otherwise peripherally hyper-activate the brain's microglia ${ }^{187-189}$. This is supported by emerging evidence from multiple experimental models further suggests that probiotic supplementation may have protective role against LPS induced neurodegeneration ${ }^{178,179}$. Even in the absence of TBI or endotoxemia, the host microbiota inhabiting the gut play a critical role in the maturation and function of the microglia in the brain ${ }^{89}$.

Recent studies provide further support of this novel approach of healing the gut to treat the brain. Using an experimental murine model to induce an inflammatory response in the gut, D'Mello and colleagues observed sickness behavior and hyperactive microglia upon ex-vivo examination of neuronal tissue relative to sham-control group. After administering either probiotics or placebo treatment to the mice, the researchers observed a significant reduction of systemic inflammation, sickness behavior, and suppression of microglial hyperactivation only in the mice that received $\operatorname{probiotics}^{177}$. 
As the appreciation of the microbiome has continued to grow, more studies are beginning to investigate the effects of probiotic supplementation in humans across different disease states. Multiple placebo-controlled, double-blind randomized control trials across various patient populations demonstrate the ability of probiotics to reduce levels of serum endotoxin and pro-inflammatory cytokines while simultaneously increasing levels of anti-inflammatory cytokines ${ }^{180-184}$. These studies make no effort to investigate the influence of these probiotics on neuronal function or health, but the clinical data from Sandiego et al illustrate that attenuating systemic inflammation will likely aid in regulating microglial activity in the brain to benefit recovery following head injury ${ }^{95}$. Human studies with a more focused interest on neuronal function and health provide further evidence for the ability of probiotics to influence the CNS through the GBMAx ${ }^{185,186}$.

Importantly, prebiotic supplementation of dietary fiber is another therapeutic arm with significant leverage. Of particular relevance, prebiotics have been found to attenuate the behavioral deficits and the pathophysiological response to LPS in multiple experimental models of endotoxemia ${ }^{190191}$.

\subsubsection{Nutritional Supplementation}

The gut microflora is also intricately involved in our ability to process nutrients, many of which influence brain health ${ }^{192}$. Docosahexaenoic acid (DHA), for example, is an omega-3 fatty acid that exhibits strong anti-inflammatory and neuroprotective effects ${ }^{193}$. In experimental models of TBI, DHA supplementation is known to reduce axonal damage in rodents ${ }^{194}$. This finding was recently supported in a clinical study of collegiate football players where prophylactic DHA supplementation significantly reduced serum levels of a known biomarker (Neurofilament Light; NFL) for axonal damage that was otherwise significantly elevated over the course of the season in athletes without 
$\mathrm{DHA}^{195}$. Notably, researchers have found that administration of probiotics augments DHA metabolism and leads to significantly greater levels of DHA in the brain to further promote its neuroprotective effects ${ }^{196,197}$. This suggests a possible synergistic effect between DHA and probiotic supplementation to optimize this GBMAx intervention to protect the brain from neurotruama.

In addition to DHA, there are many other nutritional interventions that may be viable therapies for TBI ${ }^{198-200}$. Focusing solely on fortifying the gut, there are many nutrients that may strengthen the integrity of the intestinal barrier. For example, Zinc, Vitamin D, and Magnesium are all known to improve the health of the intestinal mucosa lining ${ }^{201-203}$. Interestingly, all of these nutrients have been shown to improve outcomes following neurotraumatic events, but these studies focus on alternative mechanisms. Further research is needed to understand the influence of nutritional supplementation as a neuroprotective strategy.

\subsubsection{Vagus Nerve Stimulation}

A complete understanding of the GBMAx following head injuries is not possible without proper consideration of the vagus nerve. The vagus nerve mediates much of the GBMAx communication and may be significantly implicated in this positive feedback loop of inflammation ${ }^{204}$. Remarkably, vagotomised rats, do not experience sickness behavior following LPS injection ${ }^{205}$ and experimental models have demonstrated that vagus nerve stimulation (VNS) is an effective therapy for attenuating inflammation triggered by endotoxemia ${ }^{206}$. In addition to promoting an anti-inflammatory cholinergic response, VNS also directly improves the integrity of the tight gap junction proteins that regulate intestinal permeability ${ }^{207}$. VNS has also been shown to decrease intestinal permeability and improve the integrity of the blood-brain barrier, both of which would be beneficial following $\mathrm{TBI}^{208,209}$. 
Indeed, preclinical data directly demonstrates the ability of VNS to be protective when applied both prophylactically before TBI and as a therapeutic tool when performed during the recovery process. As a prophylactic intervention, VNS was shown to prevent neurotrauma induced intestinal permeability in a model of experimental $\mathrm{TBI}^{111}$. Other early preclinical studies investigating the therapeutic effects of VNS following head injuries demonstrate that it improves motor and cognitive outcomes while also reducing secondary neuronal damage ${ }^{210}$. Given the mounting evidence in support of the emerging hypothesis that neurodegenerative pathology may start in the gut before spreading to the CNS via the vagus nerve, the bidirectional involvement of the GBMAx and the vagus nerve in the pathophysiological response to TBI warrants further investigation ${ }^{211}$.

\subsection{4: Gut-derived Neuropeptides}

Ghrelin is a orexigenic hormone produced in the gut that functions as a neuropeptide in the CNS. In murine models of endotoxemia (experimentally induced sepsis via cecal ligation and puncture), studies have demonstrated that ghrelin is effective at ameliorating intestinal dysfunction and downregulating the subsequent systemic pro-inflammatory immune response $\mathrm{e}^{212,213}$. Researchers report that this effect is likely mediated through vagus nerve stimulation as mice that received a vagotomy did not respond to ghrelin ${ }^{212}$. The effects of ghrelin have been directly investigated as a therapeutic intervention for neurotrauma induced intestinal dysfunction in pre-clinical trials. In a weight-drop model of experimental TBI, ghrelin administered immediately before and after TBI was found to prevent 1) physical damage to intestinal tissue, 2) intestinal permeability, and 3) systemic inflammatory response. Ghrelin led to a significant suppression of MLCK (a marker of injury to intestinal epithelial tissue) and blinded pathologist only reported physical damage to intestinal tissue 
in the TBI group, not in the TBI+Ghrelin group or the sham group ${ }^{214}$. In a separate study, an experimental model of Intracerebral Hemorhage (ICH) produced consistent results even when ghrelin was only administered post-injury. In this study, ghrelin attenuated markers of damaged intestinal tissue, increased intestinal permeability, and abnormal tight-junction related protein expression that was otherwise significantly different in the sham and ICH groups ${ }^{215}$. Both survival rate and measures of weight loss were significantly improved in the $\mathrm{ICH}+$ ghrelin group relative to the $\mathrm{ICH}$ group.

\section{3: Translational Challenges}

As noted in prior reviews, translating TBI from bench to bedside has unique challenges. Chief among them are 1) there is no reliable and objective method for diagnosis of TBI, particularly, mild TBI, where neuronal damage may not be readily detectable with current tools, 2) many TBIs go undiagnosed and unreported, so it may be difficult to find a true 'healthy control', and 3) although TBIs are 'acute' injuries, it is challenging to follow subjects longitudinally and control for many confounding variables between injury and the potential latent neurological symptoms ${ }^{216}$. Additionally, there are inherent challenges in translating findings from experimental animal models to human applications, and these are multiplied when investigating the gut microflora. The chief concern in this regard is that the animals are housed in such sterile environments with no true exposure to the bacterial of the external environment that humans gut microflora is exposed to and influenced $\mathrm{by}^{217}$.

Specific to our conceptual model, there are additional challenges that we foresee. 
1) Most real-world TBI occurs with comorbid polytrauma: It may be challenging to distinguish the cause of gut alterations and systemic inflammation between either ANS signaling or direct trauma to the intestines.

2) Controlling for the plasticity of the gut microflora in sham procedures: The microflora is adaptive and responsive to stress, which may cause alterations to the gut microflora even in the supposed sham control group.

3) With this integrative model across multiple physiological systems, it will be difficult to delineate the exact mechanisms of action for proposed interventions. For example, if prophylactic intake of prebiotics and probiotics are taken to 'fortify' the gut in a population of athletes, is the potential neuroprotective effect mediated by enhanced intestinal barrier function, altered metabolomics profile of SCFAs, improved glycemic control, or some other mechanism?

4) In human trials, it may be hard to distinguish the cause of post-TBI infection between intestinal dysfunction mediated bacterial translocation and immunosuppression. However, the pre-clinical data above provide sufficient evidence to suggest that this TBI-induced endotoxemia is, at least partially, mediated by intestinal dysfunction. More sensitive measures of intestinal dysfunction and intestinal permeability would be helpful.

\section{Conclusion}

There is extensive evidence highlighting the involvement of secondary injury mechanisms that may occur in the brain following repetitive or singular neurotraumatic events. Chief among this perpetual disruption to the CNS is microglial mediated neuroinflammation, which is known to increase the risk 
of numerous neuropsychiatric diseases later in life ranging from depression to CTE. There is compelling evidence to suggest that the GBMAx is a nexus for neurotrauma, inflammation, and latent disease. Data from preclinical and clinical trials demonstrate that systemic inflammation can peripherally activate microglial cells in the brain, which become harmful when hyperactivated. A prototypical systemic immune response capable of such microglial activation is observed following experimental endotoxemia, which mimics the body's response when the intestinal barrier becomes compromised. Remarkably, neurotrauma can damage the intestinal lining of the gut in a manner that achieves this same effect and produces a systemic immune response capable of exacerbating sensitive microglia in the brain that are previously primed from the impact forces of the initial head injury. Therefore, we posit that GBMAx is a viable target for novel therapies aimed at suppressing the secondary injury mechanisms following neurotrauma to 1) improve immediate outcome (e.g., reduce risk of PCS) and 2) reduce risk of latent neurological disease. The pleiotropic effects of the GBMAx across multiple domains of human physiology is still relatively unclear and it likely mediates CNS health through varied mechanisms. However, we believe the evidence outlined herein provides sufficient support to further investigate the role of the GBMAx in recovery from TBI and in the mechanisms that mediate risk of latent disease. Therapeutically, interventions could be created to fulfill a healing capacity following injury or they could be more effective as a prophylactic strategy for neuroprotection. Prophylactically, could measures that fortify the intestinal barrier improve brain resilience amongst those with high exposure to neurotrauma? Similarly, could the same approach improve the brain's resilience by reducing inflammation in order to reduce the risk of neurodegeneration amongst populations with significant history of neurotrauma? Clearly, more work is needed to answer these questions, but we believe the conceptual model proposed in this review lays a foundation to further investigate TBI through this integrative lens. 


\section{Funding}

There are no external funding sources for this work

\section{Conflicts of Interest}

The authors declare no conflicts of interest.

\section{References}

1. Humphreys, I., Wood, R. L., Phillips, C. J. \& Macey, S. The costs of traumatic brain injury: A literature review. Clin. Outcomes Res. 5, 281-287 (2013).

2. Iverson, G. L., Gardner, A. J., McCrory, P., Zafonte, R. \& Castellani, R. J. A critical review of chronic traumatic encephalopathy. Neurosci. Biobehav. Rev. 56, 276-293 (2015).

3. Sundman, M. H., Hall, E. E. \& Chen, N.-K. Examining the Relationship between Head Trauma and Neurodegenerative Disease: A Review of Epidemiology, Pathology and Neuroimaging Techniques. J. Alzheimer's Dis. Park. 4, 1-21 (2014).

4. Holsinger, T. et al. Head injury in early adulthood and the lifetime risk of depression. Arch. Gen. Psychiatry 59, 17-22 (2002).

5. Gavett, B. E., Stern, R. A., Cantu, R. C., Nowinski, C. J. \& McKee, A. C. Mild traumatic brain injury: a risk factor for neurodegeneration. Alzheimers.Res.Ther. 2, 18 (2010).

6. Faul, M., Xu, L., Wald, M. M. \& Coronado, V. G. Traumatic Brain Injury in the United States: Emergency Department Visits, Hospitilizations and Deaths 2002-2006. Atlanta (GA): Centers for Disease Control and Prevention, National Center for Injury Prevention and Control (2010).

7. Rusnak, M. Traumatic brain injury: Giving voice to a silent epidemic. Nat. Publ. Gr. 9, 186187 (2013).

8. Dashnaw, M. L., Petraglia, A. L. \& Bailes, J. E. An overview of the basic science of concussion and subconcussion: where we are and where we are going. Neurosurg. Focus 33, E5: 1-9 (2012).

9. Masel, B. E. \& DeWitt, D. S. Traumatic brain injury: a disease process, not an event. J. Neurotrauma 27, 1529-1540 (2010).

10. Romijn, J. A., Corssmit, E. P., Havekes, L. M. \& Pijl, H. Gut???brain axis. Curr. Opin. Clin. Nutr. Metab. Care 11, 518-521 (2008).

11. Cryan, J. F. \& Dinan, T. G. Mind-altering microorganisms: the impact of the gut microbiota on brain and behaviour. Nat. Rev. Neurosci. 13, 701-712 (2012).

12. Dinan, T. G. \& Cryan, J. F. Gut-brain axis in 2016: Brain-gut-microbiota axis - mood, metabolism and behaviour. Nat. Rev. Gastroenterol. Hepatol. (2017). doi:10.1038/nrgastro.2016.200

13. Sender, R., Fuchs, S. \& Milo, R. Revised Estimates for the Number of Human and Bacteria Cells in the Body. PLoS Biol. 14, 1-14 (2016).

14. Kelly, J. R. et al. Breaking down the barriers: the gut microbiome, intestinal permeability and stress-related psychiatric disorders. Front. Cell. Neurosci. 9, 392 (2015). 
15. Bengmark, S. Gut microbiota, immune development and function. Pharmacol. Res. 69, 87113 (2013).

16. Sonnenburg, J. L. \& Fischbach, M. A. Community health care: therapeutic opportunities in the human microbiome. Sci Transl Med 3, 78ps12 (2011).

17. Mayer, E. a, Knight, R., Mazmanian, S. K., Cryan, J. F. \& Tillisch, K. Gut microbes and the brain: paradigm shift in neuroscience. J. Neurosci. 34, 15490-6 (2014).

18. Montiel-Castro, A. J., González-Cervantes, R. M., Bravo-Ruiseco, G. \& Pacheco-López, G. The microbiota-gut-brain axis: neurobehavioral correlates, health and sociality. Front. Integr. Neurosci. 7, 70 (2013).

19. Mayer, E. a, Tillisch, K. \& Gupta, A. Gut / brain axis and the microbiota. J. Clin. Invest. 125, 926-938 (2015).

20. Turner, J. R. Intestinal mucosal barrier function in health and disease. Nat. Rev. Immunol. 9, 799-809 (2009).

21. Farhadi, A., Banan, A., Fields, J. \& Keshavarzian, A. Intestinal barrier: An interface between health and disease. J. Gastroenterol. Hepatol. 18, 479-497 (2003).

22. Gallo, R. L. \& Hooper, L. V. Epithelial antimicrobial defence of the skin and intestine. Nat. Rev. Immunol. 12, 503-516 (2012).

23. Ohland, C. L. \& Macnaughton, W. K. Probiotic bacteria and intestinal epithelial barrier function. Am. J. Gastrointest. Liver Physiol. 298, G807-819 (2010).

24. Kharrazian, D. Traumatic brain injury and the effect on the brain-gut axis. Altern. Ther. Health Med. 21, 28-32 (2015).

25. Hang, C. H., Shi, J. X., Li, J. S., Wu, W. \& Yin, H. X. Alterations of intestinal mucosa structure and barrier function following traumatic brain injury in rats. World J Gastroenterol 9, 2776-2781 (2003).

26. Bansal, V. et al. Traumatic Brain Injury and Intestinal Dysfunction: Uncovering the NeuroEnteric Axis. J. Neurotrauma 26, 110306202455053 (2009).

27. Kumar, A. \& Loane, D. J. Neuroinflammation after traumatic brain injury: Opportunities for therapeutic intervention. Brain. Behav. Immun. 26, 1191-1201 (2012).

28. Witcher, K. G., Eiferman, D. S. \& Godbout, J. P. Priming the Inflammatory Pump of the CNS after Traumatic Brain Injury. Trends Neurosci. 38, 609-620 (2015).

29. Norden, D. M., Muccigrosso, M. M. \& Godbout, J. P. Microglial priming and enhanced reactivity to secondary insult in aging, and traumatic CNS injury, and neurodegenerative disease. Neuropharmacology 96, 29-41 (2015).

30. Najjar, S., Pearlman, D. M., Alper, K., Najjar, A. \& Devinsky, O. Neuroinflammation and psychiatric illness. J. Neuroinflammation 10, 1-24 (2013).

31. Perry, V. H. \& Holmes, C. Microglial priming in neurodegenerative disease. Nat. Rev. Neurol. 10, 217-24 (2014).

32. Anthony, D. C., Couch, Y., Losey, P.\& Evans, M. C. The systemic response to brain injury and disease. Brain. Behav. Immun. 26, 534-540 (2012).

33. Bischoff, S. C. et al. Intestinal permeability - a new target for disease prevention and therapy. Gastroenterology 14, 1-25 (2014).

34. Arrieta, M. C., Bistritz, L. \& Meddings, J. B. Alterations in intestinal permeability. Gut 55, 1512-1520 (2006).

35. Cunningham, C. Microglia and neurodegeneration: The role of systemic inflammation. Glia 61, 71-90 (2013).

36. Palin, K., Cunningham, C., Forse, P., Perry, V. H. \& Platt, N. Systemic inflammation 
switches the inflammatory cytokine profile in CNS Wallerian degeneration. Neurobiol. Dis. 30, 19-29 (2008).

37. Menon, D. K., Schwab, K., Wright, D. W. \& Maas, A. I. Position statement: Definition of traumatic brain injury. Arch. Phys. Med. Rehabil. 91, 1637-1640 (2010).

38. Ruff, R. M., Iverson, G. L., Barth, J. T., Bush, S. S. \& Broshek, D. K. Recommendations for diagnosing a mild traumatic brain injury: A national academy of neuropsychology education paper. Arch. Clin. Neuropsychol. 24, 3-10 (2009).

39. King, N. S. Post-concussion syndrome: Clarity amid the controversy? British Journal of Psychiatry 183, 276-278 (2003).

40. Bailes, J. E., Petraglia, A. L., Omalu, B. I., Nauman, E. \& Talavage, T. Role of subconcussion in repetitive mild traumatic brain injury. J. Neurosurg. 119, 1235-45 (2013).

41. Crisco, J. J. et al. Head impact exposure in collegiate football players. J. Biomech. 44, 26732678 (2011).

42. Echlin, P. S. et al. A prospective study of physician-observed concussion during a varsity university ice hockey season: incidence and neuropsychological changes. Part 2 of 4. Neurosurg Focus 33, E2 1-11 (2012).

43. Koerte, I. K., Ertl-wagner, B., Reiser, M., Zafonte, R. \& Shenton, M. E. White Matter Integrity in the Brains of Professional. JAMA 308, 1-6 (2013).

44. Davenport, E. M. et al. Subconcussive impacts and imaging findings over a season of contact sports. Concussion (2016). doi:10.2217/cnc-2016-0003

45. Sharma, D. et al. Subconcussive Head Impact Exposure and White Matter Tract Changes over a Single Season of Youth Football. Radiology 281, 919-926 (2017).

46. Chen, X. H., Johnson, V. E., Uryu, K., Trojanowski, J. Q. \& Smith, D. H. A lack of amyloid ?? plaques despite persistent accumulation of amyloid ?? in axons of long-term survivors of traumatic brain injury. Brain Pathol. 19, 214-223 (2009).

47. Johnson, V. E. et al. Inflammation and white matter degeneration persist for years after a single traumatic brain injury. Brain 136, 28-42 (2013).

48. Mouzon, B. C. et al. Chronic neuropathological and neurobehavioral changes in a repetitive mild traumatic brain injury model. Ann. Neurol. 75, 241-254 (2014).

49. DiSabato, D. J., Quan, N. \& Godbout, J. P. Neuroinflammation: the devil is in the details. J. Neurochem. 139, 136-153 (2016).

50. Aamodt, S. Focus on glia and disease. Nat. Neurosci. 10, 2007 (2007).

51. Bramlett, H. \& Dietrich, W. D. Long-Term Consequences of Traumatic Brain Injury: Current Status of Potential Mechanisms of Injury and Neurologic Outcomes. J. Neurotrauma 33136, $1-62$ (2014).

52. Chen, Z. \& Trapp, B. D. Microglia and neuroprotection. J. Neurochem. 136, 10-17 (2016).

53. Smith, C. Review: The long-term consequences of microglial activation following acute traumatic brain injury. Neuropathology and Applied Neurobiology 39, 35-44 (2013).

54. Smith, D. H., Johnson, V. E. \& Stewart, W. Chronic neuropathologies of single and repetitive TBI: substrates of dementia? Nat. Rev. Neurol. 9, 211-21 (2013).

55. Aungst, S. L., Kabadi, S. V, Thompson, S. M., Stoica, B. A. \& Faden, A. I. Repeated mild traumatic brain injury causes chronic neuroinflammation, changes in hippocampal synaptic plasticity, and associated cognitive deficits. J. Cereb. Blood Flow Metab. 34, 1223-32 (2014).

56. Fenn, A. M. et al. Immune activation promotes depression 1 month after diffuse brain injury: A role for primed microglia. Biol. Psychiatry 76, 575-584 (2014). 
57. Shitaka, Y. et al. Repetitive closed-skull traumatic brain injury in mice causes persistent multifocal axonal injury and microglial reactivity. J. Neuropathol. Exp. Neurol. 70, 551-67 (2011).

58. Weil, Z. M., Gaier, K. R. \& Karelina, K. Injury timing alters metabolic, inflammatory and functional outcomes following repeated mild traumatic brain injury. Neurobiol. Dis. 70, 108116 (2014).

59. Brooks, W. M. et al. Metabolic and cognitive response to human traumatic brain injury: a quantitative proton magnetic resonance study. J Neurotrauma 17, 629-640 (2000).

60. Johnson, V. E., Stewart, W. \& Smith, D. H. Axonal pathology in traumatic brain injury. Exp. Neurol. 246, 35-43 (2013).

61. Ramlackhansingh, A. F. et al. Inflammation after trauma: Microglial activation and traumatic brain injury. Ann. Neurol. 70, 374-383 (2011).

62. Coughlin, J. M. et al. Neuroinflammation and brain atrophy in former NFL players: An in vivo multimodal imaging pilot study. Neurobiol. Dis. 74, 58-65 (2015).

63. Coughlin, J. M. et al. Imaging of Glial Cell Activation and White Matter Integrity in Brains of Active and Recently Retired National Football League Players. JAMA Neurol. 6, 193-201 (2016).

64. Koerte, I. K. et al. Altered neurochemistry in former professional soccer players without a history of concussion. J. Neurotrauma 1293, online first (2015).

65. Lull, M. E. \& Block, M. L. Microglial Activation and Chronic Neurodegeneration. Neurotherapeutics 7, 354-365 (2010).

66. Wachholz, S. et al. Microglia activation is associated with IFN- $\alpha$ induced depressive-like behavior. Brain. Behav. Immun. 55, 105-113 (2016).

67. Simon, D. W. et al. The far-reaching scope of neuroinflammation after traumatic brain injury. Nat. Rev. Neurol. 13, 171-191 (2017).

68. Manuscript, A. \& Endotoxins, L. NIH Public Access. October 71, 1-57 (2008).

69. Sandiego, C. M. et al. Imaging robust microglial activation after lipopolysaccharide administration in humans with PET. Proc. Natl. Acad. Sci. U. S. A. 112, 12468-73 (2015).

70. Kitazawa, M. Lipopolysaccharide-Induced Inflammation Exacerbates Tau Pathology by a Cyclin-Dependent Kinase 5-Mediated Pathway in a Transgenic Model of Alzheimer's Disease. J. Neurosci. 25, 8843-8853 (2005).

71. Lasselin, J. et al. Mood disturbance during experimental endotoxemia: Predictors of state anxiety as a psychological component of sickness behavior. Brain. Behav. Immun. 57, 30-37 (2016).

72. Slyepchenko, A. et al. Gut Microbiota, Bacterial Translocation, and Interactions with Diet: Pathophysiological Links between Major Depressive Disorder and Non-Communicable Medical Comorbidities. Psychother Psychosom 8686, 31-4631 (2017).

73. Forsyth, C. B. et al. Increased intestinal permeability correlates with sigmoid mucosa alphasynuclein staining and endotoxin exposure markers in early Parkinson's disease. PLoS One 6, e28032 (2011).

74. Hornig, M. The role of microbes and autoimmunity in the pathogenesis of neuropsychiatric illness. Curr. Opin. Rheumatol. 25, 488-795 (2013).

75. Sampson, T. R. et al. Gut Microbiota Regulate Motor Deficits and Neuroinflammation in a Model of Parkinson's Disease. Cell 167, 1469-1480.e12 (2016).

76. Reddy, B. L. \& Saier, M. H. Autism and our intestinal microbiota. J. Mol. Microbiol. Biotechnol. 25, 51-55 (2015). 
77. Balzola, F., Bernstein, C., Ho, G. T. \& Lees, C. A human gut microbial gene catalogue established by metagenomic sequencing: Commentary. Inflamm. Bowel Dis. Monit. 11, 28 (2010).

78. Gill, S. et al. Metagenomic analysis of the human distal gut microbiome. Science (80-. ). 312, 1355 (2006).

79. Blaser, M. Stop the killing of beneficial bacteria. Nature 476, 393-394 (2011).

80. Gronlund, M. M., Lehtonen, O. P., Eerola, E. \& Kero, P. Fecal microflora in healthy infants born by different methods of delivery: permanent changes in intestinal flora after cesarean delivery. J Pediatr Gastroenterol Nutr 28, 19-25 (1999).

81. De Filippo, C. et al. Impact of diet in shaping gut microbiota revealed by a comparative study in children from Europe and rural Africa. Proc. Natl. Acad. Sci. U. S. A. 107, 14691-6 (2010).

82. Vuong, H. E., Yano, J. M., Fung, T. C. \& Hsiao, E. Y. The Microbiome and Host Behavior. 21-49 (2017). doi:10.1146/annurev-neuro-072116-031347

83. Zhou, L. \& Foster, J. A. Psychobiotics and the gut???brain axis: In the pursuit of happiness. Neuropsychiatr. Dis. Treat. 11, 715-723 (2015).

84. $\mathrm{Xu}, \mathrm{M}$. Q. et al. Fecal microbiota transplantation broadening its application beyond intestinal disorders. World J. Gastroenterol. 21, 102-111 (2015).

85. Rogers, G. B. et al. From gut dysbiosis to altered brain function and mental illness: mechanisms and pathways. Mol. Psychiatry 21, 1-11 (2016).

86. Sampson, T. R. \& Mazmanian, S. K. Control of brain development, function, and behavior by the microbiome. Cell Host Microbe 17, 565-576 (2015).

87. Sherwin, E., Rea, K., Dinan, T. G. \& Cryan, J. F. A gut (microbiome) feeling about the brain. Curr Opin Gastroenterol 32, 96-102 (2016).

88. Erny, D., Hrab?? de Angelis, A. L. \& Prinz, M. Communicating systems in the body: how microbiota and microglia cooperate. Immunology 150, 7-15 (2017).

89. Erny, D. et al. Host microbiota constantly control maturation and function of microglia in the CNS. Nat. Neurosci. 18, 965-977 (2015).

90. Rooks, M. G. \& Garrett, W. S. Gut microbiota, metabolites and host immunity. Nat. Rev. Immunol. 16, 341-52 (2016).

91. Hu, X., Wang, T. \& Jin, F. Alzheimer???s disease and gut microbiota. Science China Life Sciences 59, 1006-1023 (2016).

92. Ghaisas, S., Maher, J. \& Kanthasamy, A. Gut microbiome in health and disease: Linking the microbiome-gut-brain axis and environmental factors in the pathogenesis of systemic and neurodegenerative diseases. Pharmacol. Ther. 158, 52-62 (2016).

93. Mulak, A. \& Bonaz, B. Brain-gut-microbiota axis in Parkinson's disease. World J. Gastroenterol. 21, 10609-10620 (2015).

94. Kelly, J. R. et al. Transferring the blues: Depression-associated gut microbiota induces neurobehavioural changes in the rat. J. Psychiatr. Res. 82, 109-118 (2016).

95. Sandiego, C. M. et al. Imaging robust microglial activation after lipopolysaccharide administration in humans with PET. Proc. Natl. Acad. Sci. U. S. A. 112, 12468-73 (2015).

96. Asti, a \& Gioglio, L. Can a Bacterial Endotoxin be a Key Factor in the Kinetics of Amyloid Fibril Formation? J Alzheimers Dis 39, 169-179 (2013).

97. Lee, J. W. et al. Neuro-inflammation induced by lipopolysaccharide causes cognitive impairment through enhancement of beta-amyloid generation. J. Neuroinflammation 5, 37 (2008). 
98. Hill, J. M. \& Lukiw, W. Microbial-generated amyloids and Alzheimer's disease (AD). Front. Aging Neurosci. 7, 9 (2015).

99. Zhang, R. et al. Circulating endotoxin and systemic immune activation in sporadic amyotrophic lateral sclerosis (sALS). J. Neuroimmunol. 206, 121-124 (2009).

100. Qin, L. et al. Systemic LPS causes chronic neuroinflammation and progressive neurodegeneration. Glia 55, 453-462 (2007).

101. Perry, V. H. Contribution of systemic inflammation to chronic neurodegeneration. Acta Neuropathol. 120, 277-286 (2010).

102. Desai, M. S. et al. A Dietary Fiber-Deprived Gut Microbiota Degrades the Colonic Mucus Barrier and Enhances Pathogen Susceptibility. Cell 167, 1339-1353.e21 (2016).

103. Mobley, A. R., Jones, J. M., Rodriguez, J., Slavin, J. \& Zelman, K. M. Identifying practical solutions to meet America's fiber needs: Proceedings from the food \& fiber summit. Nutrients 6, 2540-2551 (2014).

104. Sonnenburg, E. D. \& Sonnenburg, J. L. Starving our microbial self: The deleterious consequences of a diet deficient in microbiota-accessible carbohydrates. Cell Metab. 20, 779-786 (2014).

105. Baguley, I. J. et al. The incidence of dysautonomia and its relationship with autonomic arousal following traumatic brain injury. Brain Inj. 21, 1175-1181 (2007).

106. Baguley, I. J., Heriseanu, R. E., Cameron, I. D., Nott, M. T. \& Slewa-Younan, S. A critical review of the pathophysiology of dysautonomia following traumatic brain injury. Neurocrit. Care 8, 293-300 (2008).

107. Pilitsis, J. G. \& Rengachary, S. S. Complications of head injury. Neurol Res 23, 227-236 (2001).

108. Olsen, A. B. et al. Effects of traumatic brain injury on intestinal contractility. Neurogastroenterol. Motil. 25, 593-e463 (2013).

109. Sun, B. et al. The Effects of Lactobacillus acidophilus on the Intestinal Smooth Muscle Contraction through PKC/MLCK/MLC Signaling Pathway in TBI Mouse Model. PLoS One 10, e0128214 (2015).

110. Katzenberger, R. J., Ganetzky, B. \& Wassarman, D. A. The gut reaction to traumatic brain injury. Fly (Austin). 9, 68-74 (2015).

111. Bansal, V. et al. Stimulating the central nervous system to prevent intestinal dysfunction after traumatic brain injury. J Trauma 68, 1059-1064 (2010).

112. Feighery, L. et al. Increased intestinal permeability in rats subjected to traumatic frontal lobe percussion brain injury. J Trauma 64, 131-138 (2008).

113. Santos, A., Gon??alves, P., Ara??jo, J. R. \& Martel, F. Intestinal permeability to glucose after experimental traumatic brain injury: Effect of gadopentetate dimeglumine administration. Basic Clin. Pharmacol. Toxicol. 103, 247-254 (2008).

114. Jin, W. et al. Increased intestinal inflammatory response and gut barrier dysfunction in Nrf2deficient mice after traumatic brain injury. Cytokine 44, 135-140 (2008).

115. Hu, Y. C. et al. Expression of intestinal CD40 after experimental traumatic brain injury in rats. J Surg Res 184, 1022-1027 (2013).

116. Hang, C. H. et al. Effect of systemic LPS injection on cortical NF-kappaB activity and inflammatory response following traumatic brain injury in rats. Brain Res 1026, 23-32 (2004).

117. Hang, C. H., Shi, J. X., Li, J. S., Li, W. Q. \& Yin, H. X. Up-regulation of intestinal nuclear factor kappa B and intercellular adhesion molecule-1 following traumatic brain injury in rats. 
World J Gastroenterol 11, 1149-1154 (2005).

118. Zhang, X. \& Jiang, X. Effects of enteral nutrition on the barrier function of the intestinal mucosa and dopamine receptor expression in rats with traumatic brain injury. JPEN $J$ Parenter Enter. Nutr 39, 114-123 (2015).

119. Houlden, A. et al. Brain injury induces specific changes in the caecal microbiota of mice via altered autonomic activity and mucoprotein production. Brain. Behav. Immun. 57, 10-20 (2016).

120. Bested, A. C., Logan, A. C. \& Selhub, E. M. Intestinal microbiota, probiotics and mental health: from Metchnikoff to modern advances: Part II - contemporary contextual research. Gut Pathog. 5, 3 (2013).

121. Hannestad, J. et al. Endotoxin-induced systemic inflammation activates microglia:

[11C]PBR28 positron emission tomography in nonhuman primates. Neuroimage 63, 232-239 (2012).

122. Su, S.-H. et al. Elevated C-reactive protein levels may be a predictor of persistent unfavourable symptoms in patients with mild traumatic brain injury: a preliminary study. Brain. Behav. Immun. 38, 111-7 (2014).

123. Carr, J. Postconcussion syndrome: a review. Trauma 9, 21-27 (2007).

124. Schedlowski, M., Engler, H. \& Grigoleit, J. S. Endotoxin-induced experimental systemic inflammation in humans: A model to disentangle immune-to-brain communication. Brain. Behav. Immun. 35, 1-8 (2014).

125. Rathbone, A. T. L., Tharmaradinam, S., Jiang, S., Rathbone, M. P. \& Kumbhare, D. A. A review of the neuro- and systemic inflammatory responses in post concussion symptoms: Introduction of the 'post-inflammatory brain syndrome' PIBS. Brain. Behav. Immun. 46, 116 (2015).

126. Winek, K., Meisel, A. \& Dirnagl, U. Gut microbiota impact on stroke outcome: Fad or fact? J. Cereb. Blood Flow Metab. $0271678 X 16636890$ (2016). doi:10.1177/0271678X16636890

127. Denes, A., Thornton, P., Rothwell, N. J. \& Allan, S. M. Inflammation and brain injury: Acute cerebral ischaemia, peripheral and central inflammation. Brain. Behav. Immun. 24, 708-723 (2010).

128. Anrather, J. \& Iadecola, C. Inflammation and Stroke: An Overview. Neurotherapeutics 13, 661-670 (2016).

129. Schaller, B. J., Graf, R. \& Jacobs, A. H. Pathophysiological changes of the gastrointestinal tract in ischemic stroke. Am. J. Gastroenterol. 101, 1655-1665 (2006).

130. Stanley, D. et al. Translocation and dissemination of commensal bacteria in post-stroke infection. Nat. Med. 22, (2016).

131. Tascilar, N. et al. Bacterial translocation in experimental stroke: What happens to the gut barrier? Bratislava Med. J. 111, 194-199 (2010).

132. Crapser, J. et al. Ischemic stroke induces gut permeability and enhances bacterial translocation leading to sepsis in aged mice. Aging (Albany. NY). 8, 1049-1063 (2016).

133. Balzan, S., de Almeida Quadros, C., de Cleva, R., Zilberstein, B. \& Cecconello, I. Bacterial translocation: overview of mechanisms and clinical impact. J. Gastroenterol. Hepatol. 22, 464-471 (2007).

134. Worthmann, H. et al. Lipopolysaccharide binding protein, interleukin-10, interleukin-6 and C-reactive protein blood levels in acute ischemic stroke patients with post-stroke infection. $J$. Neuroinflammation 12, 13 (2015).

135. Layer, A. M. et al. Commensal microbiota affects ischemic stroke outcome by regulating 
intestinal $\gamma \delta$ T cells. Nat. Med. 13, 720-726 (2016).

136. Singh, V. et al. Microbiota Dysbiosis Controls the Neuroinflammatory Response after Stroke. J. Neurosci. 36, 7428-7440 (2016).

137. Winek, K. et al. Depletion of Cultivatable Gut Microbiota by Broad-Spectrum Antibiotic Pretreatment Worsens Outcome After Murine Stroke. Stroke 47, 1354-1363 (2016).

138. Sun, J. et al. Clostridium butyricum attenuates cerebral ischemia/reperfusion injury in diabetic mice via modulation of gut microbiota. Brain Res. 1642, 180-188 (2016).

139. Swidsinski, A., Loening-Baucke, V., Krüger, M. \& Kirsch, S. Central Nervous System and the Colonic Bioreactor: Analysis of Colonic Microbiota in Patients with Stroke Unravels Unknown Mechanisms of the Host Defense after Brain Injury. Intest. Res. 10, 332 (2010).

140. Yin, J. et al. Dysbiosis of Gut Microbiota With Reduced Trimethylamine-N-Oxide Level in Patients With Large-Artery Atherosclerotic Stroke or Transient Ischemic Attack. J. Am. Heart Assoc. 4, 1-13 (2015).

141. Yamashiro, K. et al. Gut dysbiosis is associated with metabolism and systemic inflammation in patients with ischemic stroke. PLoS One 12, e0171521 (2017).

142. Dirnagl, U. et al. Stroke-induced immunodepression: Experimental evidence and clinical relevance. Stroke 38, 770-773 (2007).

143. Najjar, S., Pearlman, D. M., Alper, K., Najjar, A. \& Devinsky, O. Neuroinflammation and psychiatric illness. J. Neuroinflammation 10, 1-24 (2013).

144. Maes, M., Kubera, M. \& Leunis, J. C. The gut-brain barrier in major depression: intestinal mucosal dysfunction with an increased translocation of LPS from gram negative enterobacteria (leaky gut) plays a role in the inflammatory pathophysiology of depression. Neuro Endocrinol. Lett. 29, 117-124 (2008).

145. Maes, M. et al. In depression, bacterial translocation may drive inflammatory responses, oxidative and nitrosative stress (O\&NS), and autoimmune responses directed against O\&NSdamaged neoepitopes. Acta Psychiatr. Scand. 127, 344-354 (2013).

146. Leonard, B. \& Maes, M. Mechanistic explanations how cell-mediated immune activation, inflammation and oxidative and nitrosative stress pathways and their sequels and concomitants play a role in the pathophysiology of unipolar depression. Neuroscience and Biobehavioral Reviews 36, 764-785 (2012).

147. Cherry, J. D., Olschowka, J. A. \& O'Banion, M. Neuroinflammation and M2 microglia: the good, the bad, and the inflamed. J. Neuroinflammation 11, 98 (2014).

148. Cherry, J. D. et al. Microglial neuroinflammation contributes to tau accumulation in chronic traumatic encephalopathy. Acta Neuropathol. Commun. 4, 112-120 (2016).

149. Kitazawa, M., Yamasaki, T. R. \& LaFerla, F. M. Microglia as a potential bridge between the amyloid ??-peptide and tau. Ann. N. Y. Acad. Sci. 1035, 85-103 (2004).

150. ElAli, A. \& Rivest, S. Microglia in Alzheimer's disease: A multifaceted relationship. Brain. Behav. Immun. 55, 138-150 (2016).

151. Parachikova, A. et al. Inflammatory changes parallel the early stages of Alzheimer disease. Neurobiol. Aging 28, 1821-1833 (2007).

152. Perlmutter, L. S., Scott, S. A., Barr??n, E. \& Chui, H. C. MHC class II???positive microglia in human brain: Association with alzheimer lesions. J. Neurosci. Res. 33, 549-558 (1992).

153. Croisier, E., Moran, L. B., Dexter, D. T., Pearce, R. K. B. \& Graeber, M. B. Microglial inflammation in the parkinsonian substantia nigra: relationship to alpha-synuclein deposition. J. Neuroinflammation 2, 14 (2005).

154. Liao, B., Zhao, W., Beers, D. R., Henkel, J. S. \& Appel, S. H. Transformation from a 
neuroprotective to a neurotoxic microglial phenotype in a mouse model of ALS. Exp. Neurol. 237, 147-152 (2012).

155. Setiawan, E. et al. Role of translocator protein density, a marker of neuroinflammation, in the brain during major depressive episodes. JAMA Psychiatry 72, 268-275 (2015).

156. Felger, J. C. \& Lotrich, F. E. Inflammatory cytokines in depression: Neurobiological mechanisms and therapeutic implications. Neuroscience 246, 199-229 (2013).

157. Holmes, C. et al. Systemic inflammation and disease progression in Alzheimer disease. Neurology 73, 768-774 (2009).

158. Ehrhart, J. et al. Humoral factors in ALS patients during disease progression. $J$ Neuroinflammation 12, 127 (2015).

159. Engelhart, M. J. et al. Inflammatory proteins in plasma and the risk of dementia: the rotterdam study. Arch. Neurol. 61, 668-672 (2004).

160. Mogi, M. et al. Tumor necrosis factor-?? (TNF-??) increases both in the brain and in the cerebrospinal fluid from parkinsonian patients. Neurosci. Lett. 165, 208-210 (1994).

161. Song, I. U., Kim, J. S., Chung, S. W. \& Lee, K. S. Is there an association between the level of high-sensitivity C-reactive protein and idiopathic parkinson's disease? a comparison of parkinson's disease patients, disease controls and healthy individuals. Eur. Neurol. 62, 99104 (2009).

162. Gimeno, D. et al. Associations of C-reactive protein and interleukin-6 with cognitive symptoms of depression: 12-year follow-up of the Whitehall II study. Psychol. Med. 39, 41323 (2009).

163. Tan, Z. S. et al. Inflammatory markers and the risk of Alzheimer disease: The Framingham study. Neurology 68, 1902-1908 (2007).

164. Schmidt, R. et al. Early inflammation and dementia: A 25-year follow-up of the HonoluluAsia Aging Study. Ann. Neurol. 52, 168-174 (2002).

165. Seel, R. T. et al. Depression after traumatic brain injury: A National Institute on Disability and Rehabilitation Research Model Systems multicenter investigation. Arch. Phys. Med. Rehabil. 84, 177-184 (2003).

166. Kerr, Z. Y., Marshall, S. W., Harding, H. P. \& Guskiewicz, K. M. Nine-Year Risk of Depression Diagnosis Increases With Increasing Self-Reported Concussions in Retired Professional Football Players. Am. J. Sports Med. 40, 2206-2212 (2012).

167. Redelmeier, D. A. \& Hsr, M. S. CMAJ Risk of suicide after a concussion. CMAJ 188, 1-8 (2016).

168. Bigler, E. D. Traumatic brain injury, neuroimaging, and neurodegeneration. Front. Hum. Neurosci. 7, 395 (2013).

169. Bickford, P. C., Flowers, A. \& Grimmig, B. Aging leads to altered microglial function that reduces brain resiliency increasing vulnerability to neurodegenerative diseases. Exp. Gerontol. 1-5 (2017). doi:10.1016/j.exger.2017.01.027

170. Morley, W. a \& Seneff, S. Diminished brain resilience syndrome: A modern day neurological pathology of increased susceptibility to mild brain trauma, concussion, and downstream neurodegeneration. Surg. Neurol. Int. 5, 97 (2014).

171. Michielan, A. \& D'Incà, R. Intestinal Permeability in Inflammatory Bowel Disease: Pathogenesis, Clinical Evaluation, and Therapy of Leaky Gut. Mediators Inflamm. 2015, 628157 (2015).

172. Noble, E. E., Hsu, T. M. \& Kanoski, S. E. Gut to brain dysbiosis: mechanisms linking Western Diet consumption, the microbiome, and cognitive impairment. Front. Behav. 
Neurosci. 11, 9 (2017).

173. von Bernhardi, R., Eugenín-von Bernhardi, L. \& Eugenín, J. Microglial cell dysregulation in brain aging and neurodegeneration. Front. Aging Neurosci. 7, 124 (2015).

174. Kesler, S. R., Adams, H. F., Blasey, C. M. \& Bigler, E. D. Premorbid intellectual functioning, education, and brain size in traumatic brain injury: an investigation of the cognitive reserve hypothesis. Appl. Neuropsychol. 10, 153-162 (2003).

175. Ulluwishewa, D. et al. Regulation of tight junction permeability by intestinal bacteria and dietary components. J. Nutr. 141, 769-776 (2011).

176. Hsieh, C.-Y. et al. Strengthening of the intestinal epithelial tight junction by Bifidobacterium bifidum. Physiol. Rep. 3, e12327-e12327 (2015).

177. D’Mello, C. et al. Probiotics Improve Inflammation-Associated Sickness Behavior by Altering Communication between the Peripheral Immune System and the Brain. J. Neurosci. 35, 10821-30 (2015).

178. Distrutti, E. et al. Modulation of intestinal microbiota by the probiotic VSL\#3 resets brain gene expression and ameliorates the age-related deficit in LTP. PLoS One 9, e106503 (2014).

179. Divyashri, G., Krishna, G., Muralidhara \& Prapulla, S. G. Probiotic attributes, antioxidant, anti-inflammatory and neuromodulatory effects of Enterococcus faecium CFR 3003: In vitro and in vivo evidence. J. Med. Microbiol. 64, 1527-1540 (2015).

180. Mohamadshahi, M. et al. Effects of probiotic yogurt consumption on inflammatory biomarkers in patients with type 2 diabetes. BioImpacts 4, 83-88 (2014).

181. Rajkumar, H. et al. Effect of probiotic (VSL\#3) and omega-3 on lipid profile, insulin sensitivity, inflammatory markers, and gut colonization in overweight adults: A randomized, controlled trial. Mediators Inflamm. 2014, 348959 (2014).

182. Lamprecht, M. et al. Probiotic supplementation affects markers of intestinal barrier, oxidation, and inflammation in trained men; a randomized, double-blinded, placebocontrolled trial. J. Int. Soc. Sports Nutr. 9, 45 (2012).

183. Kekkonen, R. A. et al. Probiotic intervention has strain-specific anti-inflammatory effects in healthy adults. World J. Gastroenterol. 14, 2029-2036 (2008).

184. Wang, W., Tan, M., Yu, J. \& Tan, L. Role of pro-inflammatory cytokines released from microglia in Alzheimer's disease. Ann Transl Med 3, 1-15 (2015).

185. Steenbergen, L., Sellaro, R., van Hemert, S., Bosch, J. A. \& Colzato, L. S. A randomized controlled trial to test the effect of multispecies probiotics on cognitive reactivity to sad mood. Brain. Behav. Immun. 48, 258-264 (2015).

186. Tillisch, K. et al. Consumption of fermented milk product with probiotic modulates brain activity. Gastroenterology 144, 1394-401, 1401-4 (2013).

187. Lamprecht, M. et al. Probiotic supplementation affects markers of intestinal barrier, oxidation, and inflammation in trained men; a randomized, double-blinded, placebocontrolled trial. J. Int. Soc. Sports Nutr. 9, 45 (2012).

188. Rodes, L. et al. Effect of probiotics Lactobacillus and Bifidobacterium on gut-derived lipopolysaccharides and inflammatory cytokines: An in vitro study using a human colonic microbiota model. J. Microbiol. Biotechnol. 23, 518-526 (2013).

189. De Punder, K. \& Pruimboom, L. Stress induces endotoxemia and low-grade inflammation by increasing barrier permeability. Front. Immunol. 6, 223 (2015).

190. Savignac, H. M. et al. Prebiotic administration normalizes lipopolysaccharide (LPS)-induced anxiety and cortical 5-HT2A receptor and IL1-?? levels in male mice. Brain. Behav. Immun. 52, 120-131 (2016). 
191. Sherry, C. L. et al. Sickness behavior induced by endotoxin can be mitigated by the dietary soluble fiber, pectin, through up-regulation of IL-4 and Th2 polarization. Brain. Behav. Immun. 24, 631-640 (2010).

192. ??stman, E. M., Liljeberg Elmst??hl, H. G. M. \& Bj??rck, I. M. E. Inconsistency between glycemic and insulinemic responses to regular and fermented milk products. Am. J. Clin. Nutr. 74, 96-100 (2001).

193. Lukiw, W. \& Bazan, N. Docosahexaenoic acid and the aging brain. J. Nutr. 138, 2510-2514 (2008).

194. Mills, J. D., Bailes, J. E., Sedney, C. L., Hutchins, H. \& Sears, B. Omega-3 fatty acid supplementation and reduction of traumatic axonal injury in a rodent head injury model. $J$. Neurosurg. 114, 77-84 (2011).

195. Oliver, J. M. et al. Effect of docosahexaenoic acid on a biomarker of head trauma in American Football. Med. Sci. Sports Exerc. 48, 974-982 (2016).

196. Tabbaa, M., Golubic, M., Roizen, M. F. \& Bernstein, A. M. Docosahexaenoic acid, inflammation, and bacterial dysbiosis in relation to periodontal disease, inflammatory bowel disease, and the metabolic syndrome. Nutrients 5, 3299-3310 (2013).

197. Wall, R. et al. Impact of administered bifidobacterium on murine host fatty acid composition. Lipids 45, 429-436 (2010).

198. Cook, A. M., Peppard, A. \& Magnuson, B. Nutrition considerations in traumatic brain injury. Nutr Clin Pr. 23, 608-620 (2008).

199. Vonder Haar, C., Peterson, T. C., Martens, K. M. \& Hoane, M. R. Vitamins and nutrients as primary treatments in experimental brain injury: Clinical implications for nutraceutical therapies. Brain Res. 1640, 114-129 (2016).

200. Scrimgeour, A. G. \& Condlin, M. L. Nutritional treatment for traumatic brain injury. $J$. Neurotrauma 31, 989-99 (2014).

201. El-Tawil, A. M. Zinc supplementation tightens leaky gut in Crohn's disease. Inflammatory Bowel Diseases 18, (2012).

202. Raftery, T. et al. Effects of vitamin D supplementation on intestinal permeability, cathelicidin and disease markers in Crohn's disease: Results from a randomised double-blind placebo-controlled study. United Eur. Gastroenterol J 3, 294-302 (2015).

203. Nevin, K. G. \& Rajamohan, T. Beneficial effects of virgin coconut oil on lipid parameters and in vitro LDL oxidation. Clin. Biochem. 37, 830-835 (2004).

204. Thayer, J. F. \& Sternberg, E. M. Neural concomitants of immunity-Focus on the vagus nerve. Neuroimage 47, 908-910 (2009).

205. Bluthe, R. M. et al. Lipopolysaccharide induces sickness behaviour in rats by a vagal mediated mechanism. C R Acad Sci III 317, 499-503 (1994).

206. Borovikova, L. V et al. Vagus nerve stimulation attenuates the systemic inflammatory response to endotoxin. Nature 405, 458-462 (2000).

207. Costantini, T. W. et al. Efferent vagal nerve stimulation attenuates gut barrier injury after burn: modulation of intestinal occludin expression. J. Trauma 68, 1349-54-6 (2010).

208. Levy, G. et al. Vagal nerve stimulation modulates gut injury and lung permeability in trauma-hemorrhagic shock. J. Trauma Acute Care Surg. 73, 338-42; discussion 342 (2012).

209. Lopez, N. E. et al. Vagal nerve stimulation decreases blood-brain barrier disruption after traumatic brain injury. J Trauma Acute Care Surg 72, 1562-1566 (2012).

210. Neren, D. et al. Vagus Nerve Stimulation and Other Neuromodulation Methods for Treatment of Traumatic Brain Injury. Neurocrit. Care 24, 308-319 (2016). 
211. Klingelhoefer, L. \& Reichmann, H. Pathogenesis of Parkinson disease-the gut-brain axis and environmental factors. Nat. Rev. Neurol. 11, 625-36 (2015).

212. Wu, R. et al. Ghrelin down-regulates proinflammatory cytokines in sepsis through activation of the vagus nerve. Ann. Surg. 245, 480-486 (2007).

213. Wu, R. et al. Orexigenic hormone ghrelin ameliorates gut barrier dysfunction in sepsis in rats. Crit. Care Med. 37, 2421-6 (2009).

214. Bansal, V. et al. The hormone ghrelin prevents traumatic brain injury induced intestinal dysfunction. J. Neurotrauma 27, 2255-60 (2010).

215. Cheng, Y. et al. Ghrelin Attenuates Intestinal Barrier Dysfunction Following Intracerebral Hemorrhage in Mice. Int. J. Mol. Sci. 17, 2032 (2016).

216. Chen, A. J. W. \& D’Esposito, M. Traumatic Brain Injury: From Bench to Beside to Society. Neuron 66, 11-14 (2010).

217. Blaser, M., Bork, P., Fraser, C., Knight, R. \& Wang, J. The microbiome explored: recent insights and future challenges. Nat. Rev. Microbiol. 11, 213-7 (2013). 